\title{
Geographical ranges of Polish mammals against zoogeographical subdivisions of the Palaearctic
}

\author{
Bożenna GRABIŃSKA \\ Stanislaw Leszczycki Institute of Geography and Spatial Organization, Polish Academy of Sciences, 00-818 Warszawa, \\ Twarda 51/55, Poland; e-mail: b.grab@twarda.pan.pl
}

\begin{abstract}
The ranges of the 89 mammalian species occurring in Poland have been described. Most of these ranges and majority of the Polish theriofauna species are confined to the Palaearctic Realm. The main aim of this work is a detail division of each Polish mammal's range into zoogeographical units (Regions, Subregions, Provinces and Subprovinces) of the Eastern and Western Palaearctic. Taxonomically, Polish terrestrial mammals belong to 20 families. Some of the species have significantly expanded their geographical ranges, with some currently occurring on all continents. Examples are given in the paper of Polish mammal species that have become extinct over the last 50 years. The numbers of mammalian species in different parts of Palaearctic (faunistic units) are presented in diagrams. The ranges of many species exceed limits of Palaearctic and spread into Paleotropical, Neotropical Realms or the Nearctic part of Holarctic.
\end{abstract}

Key words: Palaearctic, zoogeographical division, ranges, mammalian species, Poland

\section{INTRODUCTION}

The shape and size of the areas occupied by individual species and changes in these parameters are the basis of all biogeographical reasoning. Before any conclusions are drawn, the range of a given species should be described and placed within an accepted system of zoogeographical division. The identification of the geographical range of a taxon is based on possibly complete data regarding sites inhabited by individuals of this species. Three main techniques, namely, the point, line and cartogram technique, aid in the description of a taxon's range and identification of its geographical limits (Tupikova 1969). Ranges obtained using these methods can be seen in many basic publications and atlases. However, to date, no comprehensive maps of ranges have been drawn for all vertebrate species that would reveal both the internal structure of a species' range as well as its general outline. The cartogram grid technique for drawing range maps has become increasingly popular in recent years. The maps may be based on squares, trapezes related to the grid of meridians and parallels, or biogeographical units, with administrative units as the least desirable option. Cartograms combine the benefits of line and point Maps. The biggest advantage to using a cartogram is that it can be digitised by dedicated software, which aids in interpreting the faunal and floral richness of different areas, classifying types of ranges, identifying correlations between plant and animal ranges and the spatial diversification of environmental factors, the ultimate goal being to propose regional divisions relying on floristic and faunistic criteria. Particularly interesting conclusions can be drawn by studying the association of ranges with specific territories around the globe. The end result of such analyses is the most popular and bestgrounded typology of ranges used in animal geography, whereby ranges are grouped together according to the location and course of range boundaries, termed range elements.

The term "element" has been used in biogeographical literature in a number of ways and can refer to at least seven separate categories: 1. direction elements, 2. narrowly defined 
geographical elements, 3. altitudinal elements, 4. genetic elements, 5. historical elements, 6. migration elements, and 7. ecological elements (Szafer \& Zarzycki 1972, Kornaś \& Medwecka-Kornaś 2002). The farthest-reaching conclusions regarding a fauna can be drawn when its components are grouped according to their total ranges, i.e. by identifying geographical elements in a narrow meaning of this term. The analysis of a fauna with respect to the geographical elements that the fauna is made up of is a very important task but at the same time it is difficult since it must be based on previous identification of the total ranges of all species of the fauna.

Geographical elements may be narrowly or broadly defined. A broadly defined element comprises species associated with large biogeographical units whose distinct character and a high degree of independence are obvious, such as the Mediterranean, Irano-Turanian or EuroSibero-Boreo-American (= Holarctic) regions. A broadly defined geographical element thus includes species representing various geographical distributions and can be divided into subelements and other lower-order units.

The fauna of Polish mammals has not yet been fully described in statistical terms with respect to patterns of geographical distribution. Geographical elements are distinguished by identifying the range of a species or another taxon. The identification of geographical elements may serve to trace range changes within the confines of one geographical element or to class together plants or animals with overlapping ranges.

It should be emphasised at this point that none of the mammal species found in Poland is confined to the territory of Poland. The limits of these ranges are at large distances from our borders in all directions (Gentner et al. 1961).

The main aim of the present work is to describe, in a comprehensive manner, the respective geographical ranges of all mammalian taxa (species) occurring in Poland, thus providing a basis for further zoogeographical analyses.

Another main aim of this paper is to demonstrate that the patterns of distribution of the 89 species of mammals in Poland are not accidental in zoogeographical terms and that there exists a relationship between the ranges of most species and higher-order biogeographical units. The fact that the boundaries of ranges of some species have considerably changed over the last century is also pointed out.

\section{MATERIAL AND METHODS}

The initial stage of the analysis consisted in describing the range of each species on the basis of its geographical occurrence. The respective distributions were reconstructed on the basis of literature data and atlases of distribution of mammals (Pucek 1984, Pucek \& Raczyński 1983, Mitchell-Jones et al. 1999, Corbet 1978, Corbet \& Hill 1991, Gromova \& Baranovoi 1981, Görner \& Hackethal 1987, Niethammer \& Krapp 1986, Syroechkovskii \& Rogacheva 1975, and other authors).

The next step was to assign established ranges to accepted units of zoogeographical division of the Palaearctic. The Palaearctic realm is usually divided into two regions, namely, the Western and Eastern Palaearctic. Accordingly, two separate classifications were adopted for the Western and Eastern parts.

The Western Palaearctic comprises the biogeographical provinces of Europe (Udvardy 1969). The original division was arrived at by Walter drawing floristic elements, i.e. groups of species of similar or identical geographical ranges, on a map, while Freitag subsequently modified Walter's elements to account for the distribution of terrestrial vertebrates. The resulting units (provinces) are also known as "geoelements", or groups of geographical elements (Fig. 1). The units of this division are not hierarchical and the areas of some of them are not confined to Europe. This is the case with the Turanian, Iranian, Pontic, Saharo-Sind, 
Eastern Boreal or Arctic Provinces, which reach far beyond Europe. The Western Palaearctic itself is a part of a larger unit, the Palaearctic Realm, which includes, among others, the entire territory of Poland.

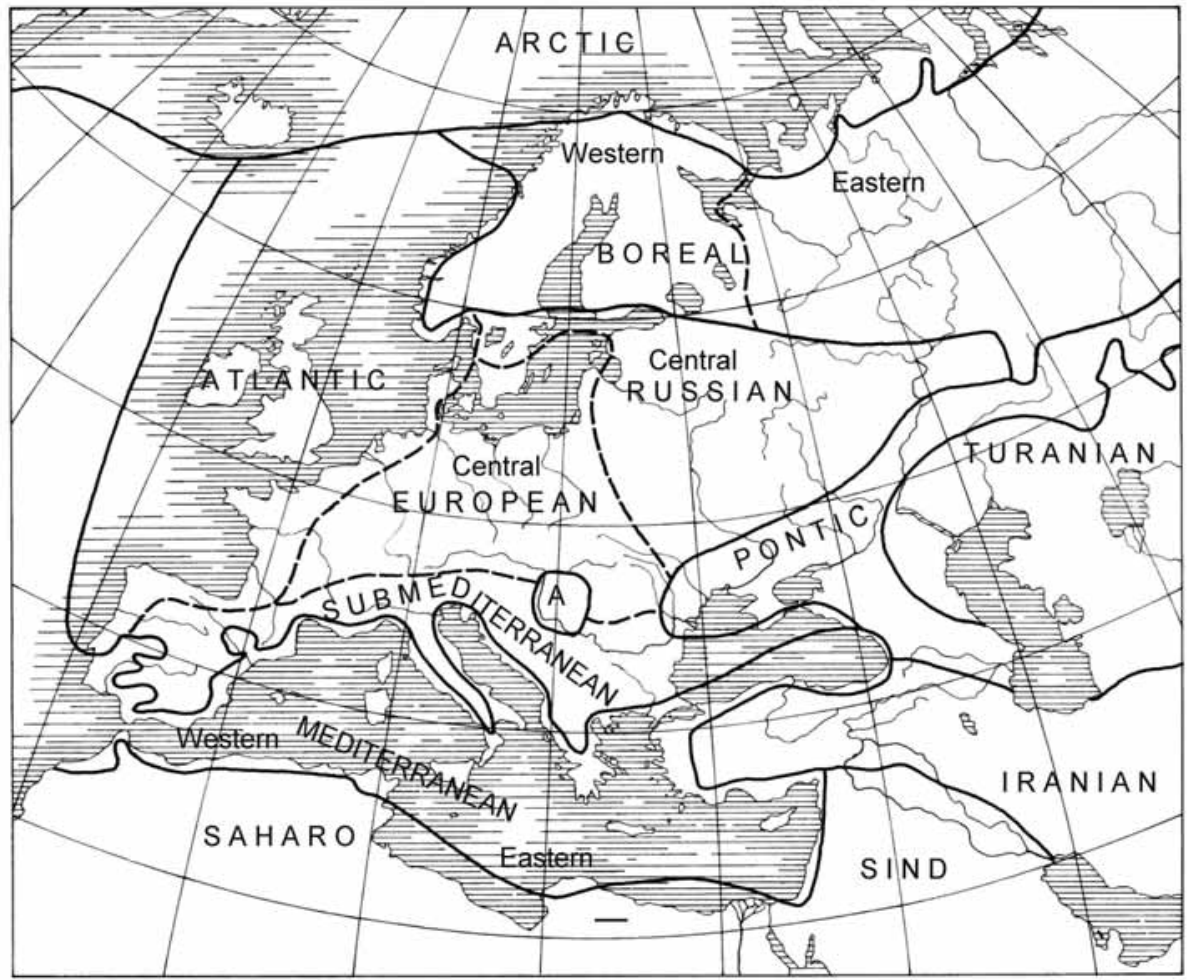

Fig. 1. Biogeographical provinces of Europe (from Freitag, 1962, following Walter 1954 (Western Palearctic); A- Pannonan endemic species.

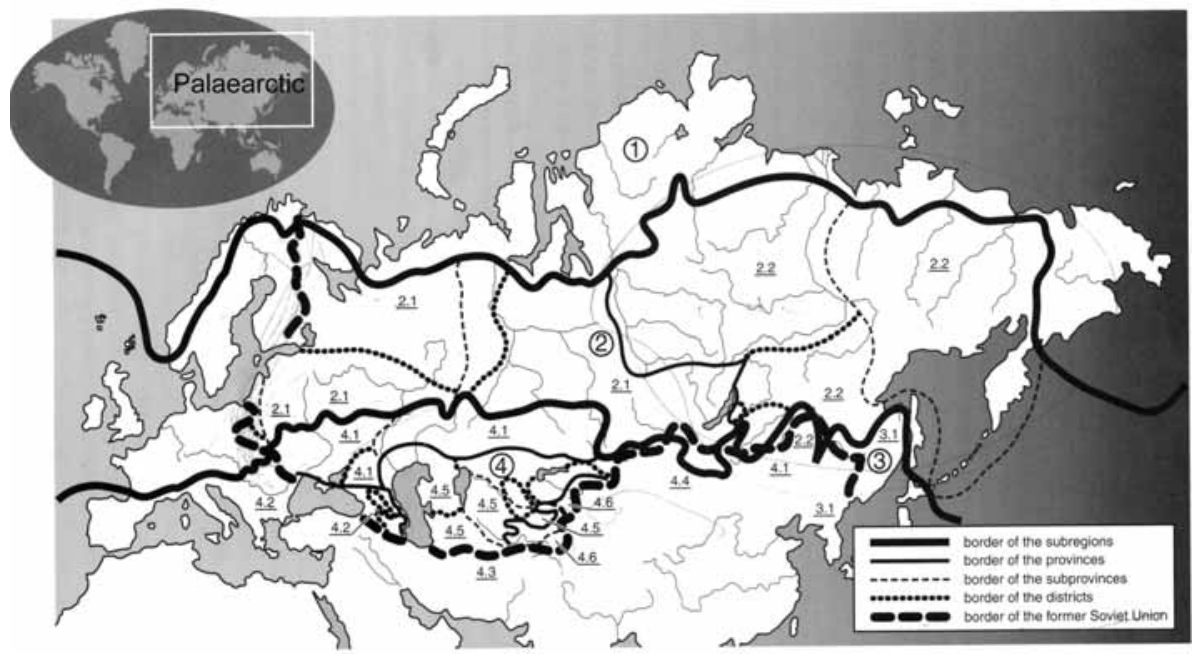

Fig. 2. Herpetogeographical Division of the Eastern Palaearctic (Szczerbak 2003). 
Table 1. Subdivisions of the Palaearctic.

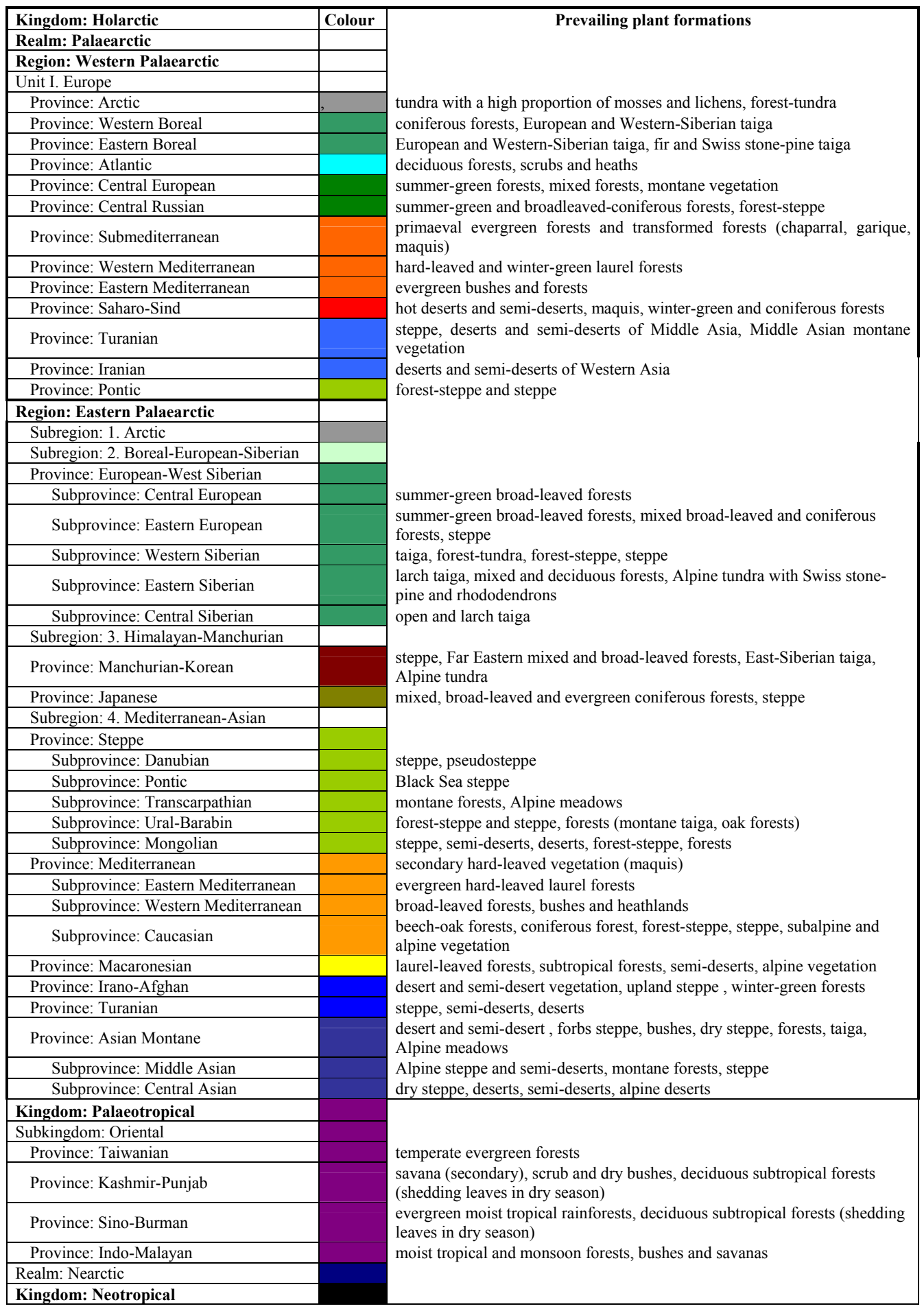


The division of the other part - the Eastern Palaearctic - was based on the distribution of reptiles (Szczerbak 2003). It must be noted that some units in this division have also been linked to the Western Palaearctic, so that they are found in both classifications (Figs 1,2 \& Table 1).

The units distinguished in the Eastern Palaearctic are arranged hierarchically.

At the topmost level of this hierarchical classification are four subregions: Arctic, BorealEuropean-Siberian, Himalayan-Manchurian and Mediterranean-Asian. 13 provinces are lowerorder units, with 15 subprovinces at the lowest level. Essential results are presented in a collective table, where each of the ranges of the 89 species of mammals is described in terms of the biogeographical units adopted for this paper (Table 2).

A colour scheme was used for the purposes of graphic representation of the ranges and their division into units with different colours used for the following provinces of the Western Palaearctic: 1. Arctic, 2. Boreal, 3. Atlantic, 4. Central European and Central Russian, 5. Submediterranean and Mediterranean, 6. Saharo-Sind and the provinces located partly in Asia: Turanian, Iranian and Pontic (Table 1).

The colour scheme for the Eastern palaearctic serves to distinguish the Arctic Subregion, the Provinces and Subprovinces of the Boreal-European-Siberian Subregion, the Provinces of the Himalayan-Manchurian Subregion, and the Provinces and steppe Subprovinces of the Mediterranean-Asian Subregion, which is further divided in the same way to mark the Mediterranean and Macaronesian Provinces. A separate colour is also used for the Asian Provinces and Subprovinces: Irano-Afghan, Turanian, Middle Asian and Central Asian.. The Provinces of the Palaeotropical Kingdom, the Nearctic Realm and the Neotropical Kingdom are also marked with separate colours (Table 1).

\section{RESULTS}

\section{The geographical ranges of Polish mammals, changes in geographical distribution and causes of these changes}

Polish mammals are classified into 6 orders and 20 families (Table 3). The most numerous orders among Polish mammals are rodents (Rodentia), bats (Chiroptera) and carnivores (Carnivora), which total 66 out of the 89 species of Polish mammals, with 22 species of rodents ${ }^{1}, 22$ species of bats $^{2}$ and 15 species of predatory mammals. The other 23 species represent the orders of insectivores (Insectivora), hares and rabbits (Lagomorpha) and eventoed ungulates (artiodactylous) (Artiodactyla).

Areas occupied by many mammalian species are subject to constant change, the dynamics of which is associated with both the natural pattern of distribution and the effect of external factors, mostly the impact of man. Range dynamics vary with time. A progressive range increases in area; a regressive range shrinks in time; a disjunctive one breaks up into smaller areas, with the possible development of relic endemics. An initially large continuous range of a species may later give rise to a number of smaller ranges as new species are formed (mutation, isolation); this is how neoendemics develop (Podbielkowski 1991).

The ranges of the species under discussion extend well beyond the area of Poland or Europe. There is only one species endemic to Poland: the Tatra pine vole (Pitymys tatricus) is endemic to the Carpathians, occurring at low numbers in the Tatra and Beskida Mts. However, even this range extends a little into the Slovakian Tatras.

\footnotetext{
${ }^{1}$ The southern birch mouse (Sicista subtilis) was not included in the statistical descriptions of contributions of individual species of rodents since this species is not recorded in Poland at present. Its occurrence requires further study.

${ }^{2}$ The soprano pipistrelle (Pipistrellus pygmaeus) was not included in the statistical descriptions of contributions of individual species due to a scarcity of data regarding its occurrence. It probably inhabits large parts of Europe, and particularly river banks and wet and marshy areas (Wołoszyn 2001).
} 
Tab. 2. Zoogeographical classification of Polish mammalian fauna; * - species whose natural ranges have changed the most significantly over the last few centuries (or decades), ${ }^{(1)}$ - western margin of its range in Poland.

\begin{tabular}{|c|c|c|c|c|c|c|c|c|c|c|c|}
\hline Family & Erina & aceidae & Talpidae & & & & Soric & cidae & & & \\
\hline Species & EREU & ERCON & TALEU S & SOARA & SOCAE & SOMINSS & SOALP & NEFOD & NEANO & CROLE & CRSUA \\
\hline No & $1 *$ & \begin{tabular}{|l|}
2 \\
\end{tabular} & 3 & 4 & \begin{tabular}{|l|}
$5^{(1)}$ \\
\end{tabular} & \begin{tabular}{l|l}
6 &
\end{tabular} & \begin{tabular}{l|l}
7 & \\
\end{tabular} & \begin{tabular}{|l|}
8 \\
\end{tabular} & 9 & 10 & 11 \\
\hline \multicolumn{12}{|l|}{ Kingdom: HOLARCTIC } \\
\hline \multicolumn{12}{|l|}{ Realm: Palaearctic } \\
\hline \multicolumn{12}{|l|}{ Region: Western Palaearctic } \\
\hline \multicolumn{12}{|l|}{ Unit: I. Europe } \\
\hline Province: Arctic & & & & $*$ & $*$ & $*$ & & $*$ & & & \\
\hline Province: Western Boreal & * & & $*$ & $*$ & & $*$ & & $*$ & & & \\
\hline Province: Eastern Boreal & & & $*$ & $*$ & $*$ & $*$ & & $*$ & & & \\
\hline Province: Atlantic & * & & $*$ & * & 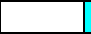 & $*$ & & $*$ & $*$ & $*$ & $*$ \\
\hline Province: Central European & * & * & * & * & $*$ & $*$ & $*$ & * & * & $*$ & $*$ \\
\hline Province: Central Russian & * & $*$ & * & * & $*$ & $*$ & 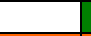 & * & 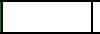 & 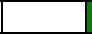 & $*$ \\
\hline Province: Submediterranean & * & $*$ & $*$ & $*$ & & $*$ & $*$ & $*$ & $*$ & $*$ & $*$ \\
\hline Province: Western Mediterranean & * & 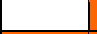 & $*$ & $*$ & & $*$ & & $*$ & $*$ & $*$ & $*$ \\
\hline Province: Eastern Mediterranean & & $*$ & $*$ & $*$ & & $*$ & & $*$ & $*$ & $*$ & $*$ \\
\hline Province: Saharo-Sind & 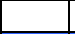 & . & & & & & & & & & $*$ \\
\hline Province: Turanian & $*$ & $*$ & & $*$ & & $*$ & & & & $*$ & $*$ \\
\hline Province: Iranian & & $*$ & & & & & & & & $*$ & $*$ \\
\hline Province: Pontic & * & $*$ & $*$ & $*$ & & $*$ & & $*$ & $*$ & $*$ & $*$ \\
\hline \multicolumn{12}{|l|}{ Region: Eastern Palearctic } \\
\hline Subregion: 1. Arctic & - & & & $*$ & $*$ & $*$ & & $*$ & & & \\
\hline Subregion: 2. Boreal-European-Siberian & * & * & * & $*$ & * & $*$ & & $*$ & & & \\
\hline Province: European-West Syberian & * & $*$ & $*$ & $*$ & $*$ & $*$ & & $*$ & - & $*$ & $*$ \\
\hline Subprovince: Central European & $*$ & $*$ & $*$ & $*$ & $*$ & $*$ & * & $*$ & * & $*$ & $*$ \\
\hline Subprovince: Eastern European & $*$ & $*$ & $*$ & $*$ & $*$ & $*$ & & $*$ & $*$ & $*$ & $*$ \\
\hline Subprovince: Western Siberian & * & $*$ & $*$ & $*$ & $*$ & $*$ & & $*$ & & & $*$ \\
\hline Subprovince: Eastern Siberian & & & & $*$ & $*$ & * & & $*$ & & - & $*$ \\
\hline Subprovince: Central Siberian & & & & $*$ & $*$ & $*$ & & $*$ & & $*$ & $*$ \\
\hline \multicolumn{12}{|l|}{ Subregion: 3. Himalayan-Manchurian } \\
\hline Province: Manchurian-Korean & & & & $*$ & $*$ & $*$ & & & & & $*$ \\
\hline Province: Japanese & & & & * & * & * & & & & & $*$ \\
\hline \multicolumn{12}{|l|}{ Subregion: 4. Mediterranean-Asian } \\
\hline \multicolumn{12}{|l|}{ Province: Steppe } \\
\hline Subprovince: Danubian & & * & $*$ & $*$ & & $*$ & & $*$ & $*$ & $*$ & $*$ \\
\hline Subprovince: Pontic & $*$ & $*$ & $*$ & $*$ & & $*$ & & $*$ & $*$ & $*$ & $*$ \\
\hline Subprovince: Transcarpathian & & $*$ & $*$ & $*$ & & $*$ & & $*$ & $*$ & $*$ & $*$ \\
\hline Subprovince: Ural-Barabin & & & & & $*$ & $*$ & & $*$ & & & $*$ \\
\hline Subprovince: Mongolian & & & * & & * & $*$ & & $*$ & & & 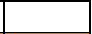 \\
\hline Province: Mediterranean & & & & & & $*$ & & $*$ & $*$ & $*$ & $*$ \\
\hline Subprovince: Eastern Mediterranean & & * & * & * & & $*$ & & $*$ & $*$ & $*$ & $*$ \\
\hline Subprovince: Western Mediterranean & * & & $*$ & $*$ & & $*$ & & $*$ & $*$ & $*$ & $*$ \\
\hline Subprovince: Caucasian & & $*$ & $*$ & * & & * & & $*$ & & & $*$ \\
\hline Province: Macaronesian & * & & & & & & & & & & \\
\hline Province: Irano-Afghan & & $*$ & & & & & & & & $*$ & $*$ \\
\hline Province:Turanian & $*$ & $*$ & & $*$ & & * & & & & $*$ & $*$ \\
\hline \multicolumn{12}{|l|}{ Province: Asian Montane } \\
\hline \multicolumn{12}{|l|}{ Subprovine: Middle Asian } \\
\hline Subprovince:Central Asian & & & $*$ & & $*$ & * & & & & $*$ & $*$ \\
\hline \multicolumn{12}{|l|}{ Kingdom: PALEOTROPICAL } \\
\hline \multicolumn{12}{|l|}{ Subkingdom:Oriental } \\
\hline \multicolumn{12}{|l|}{ Province: Taiwanian } \\
\hline \multicolumn{12}{|l|}{ Province: Kashmir-Punjab } \\
\hline \multicolumn{12}{|l|}{ Province: Sino-Burman } \\
\hline Province: Indo-Malayan & & & & & & & & & & & \\
\hline Realm: Nearctic & & & & & & & & & & & \\
\hline Kingdom: NEOTROPICAL & & & & & & & & & & & \\
\hline
\end{tabular}


Tab. 2 (continuation). Zoogeographical classification of Polish mammalian fauna; ${ }^{(2)}$ - northern edge of its range in Poland, ${ }^{(3)}$ - north-eastern periphery of its range in Poland, ${ }^{(4)}$ - northern edge of its range in Poland.

\begin{tabular}{|c|c|c|c|c|c|c|c|c|c|c|c|}
\hline \multirow{2}{*}{$\begin{array}{l}\text { Family } \\
\text { Species }\end{array}$} & \multicolumn{2}{|c|}{ Rhinolophidae } & \multicolumn{9}{|c|}{ Vespertilionidae } \\
\hline & RHH & RHF & MYM & MBE & MYN & MEM & MYS & MYB & MDS & MDA & VMU \\
\hline No & $12^{(2)}$ & 13 & 14 & $15^{(3)}$ & 16 & $17^{(4)}$ & 18 & 19 & 20 & 21 & 22 \\
\hline \multicolumn{12}{|l|}{ Kingdom: Holarctic } \\
\hline \multicolumn{12}{|l|}{ Realm: Palaearctic } \\
\hline \multicolumn{12}{|l|}{ Region: Western Palaearctic } \\
\hline \multicolumn{12}{|l|}{ Unit: I. Europe } \\
\hline \multicolumn{12}{|l|}{ Province: Arctic } \\
\hline Province: Western Boreal & & & & & * & & * & * & $*$ & $*$ & * \\
\hline Province: Eastern Boreal & & & & & $*$ & & $*$ & $*$ & $*$ & $*$ & $*$ \\
\hline Province: Atlantic & $*$ & $*$ & $*$ & $*$ & $*$ & $*$ & * & $*$ & $*$ & $*$ & $*$ \\
\hline Province: Central European & $*$ & $*$ & * & * & * & $*$ & * & $*$ & $*$ & * & * \\
\hline Province: Central Russian & 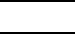 & 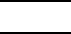 & 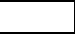 & 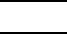 & * & 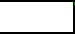 & * & * & * & * & * \\
\hline Province: Submediterranean & $*$ & $*$ & * & * & * & $*$ & $*$ & $*$ & & $*$ & * \\
\hline Province: Western Mediterranean & $*$ & $*$ & $*$ & $*$ & * & $*$ & $*$ & & & $*$ & . \\
\hline Province: Eastern Mediterranean & $*$ & $*$ & $*$ & * & * & $*$ & $*$ & & & $*$ & $*$ \\
\hline Province: Saharo-Sind & $*$ & $*$ & * & , & $*$ & $*$ & $*$ & & & $*$ & 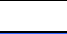 \\
\hline Province: Turanian & $*$ & $*$ & 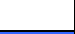 & 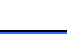 & $*$ & $*$ & $*$ & & & $*$ & $*$ \\
\hline Province: Iranian & $*$ & $*$ & * & * & $*$ & $*$ & $*$ & & & $*$ & $*$ \\
\hline Province: Pontic & $*$ & $*$ & * & * & $*$ & $*$ & $*$ & $*$ & $*$ & $*$ & $*$ \\
\hline \multicolumn{12}{|l|}{ Region: Eastern Palearctic } \\
\hline \multicolumn{12}{|l|}{ Subregion: 1. Arctic } \\
\hline Subregion: 2. Boreal-European-Siberian & & & & & & & * & * & * & * & * \\
\hline Province: European-West Siberian & . & & & & * & . & * & $*$ & $*$ & $*$ & $*$ \\
\hline Subprovince: Central European & * & * & * & * & * & * & * & $*$ & $*$ & $*$ & $*$ \\
\hline Subprovince: Eastern European & * & $*$ & * & * & $*$ & $*$ & $*$ & $*$ & $*$ & $*$ & $*$ \\
\hline Subprovince: Western Siberian & & & & & * & & $*$ & $*$ & $*$ & $*$ & $*$ \\
\hline Subprovince: Eastern Siberian & & & & & & & * & $*$ & $*$ & $*$ & $*$ \\
\hline Subprovince: Central Siberian & & & & & & & $*$ & $*$ & $*$ & $*$ & $*$ \\
\hline Subregion: 3. Himalayan-Manchurian & & . & & & * & & 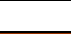 & 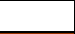 & $*$ & $*$ & $*$ \\
\hline Province: Manchurian-Korean & & $*$ & & & 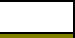 & & $*$ & $*$ & & $*$ & $*$ \\
\hline Province: Japanese & & * & & & * & & * & * & & * & * \\
\hline \multicolumn{12}{|l|}{ Subregion: 4. Mediterranean-Asian } \\
\hline \multicolumn{12}{|l|}{ Province: Steppe } \\
\hline Subprovince: Danubian & $*$ & $*$ & $*$ & $*$ & $*$ & $*$ & $*$ & $*$ & & $*$ & $*$ \\
\hline Subprovince: Pontic & $*$ & $*$ & $*$ & $*$ & $*$ & $*$ & $*$ & $*$ & $*$ & $*$ & $*$ \\
\hline Subprovince: Transcarpathian & $*$ & $*$ & $*$ & $*$ & $*$ & $*$ & $*$ & $*$ & & $*$ & $*$ \\
\hline Subprovince: Ural-Barabin & & $*$ & & & & & $*$ & $*$ & & & \\
\hline Subprovince: Mongolian & & & & & & & & $*$ & & 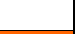 & \\
\hline Province: Mediterranean & $*$ & $*$ & $*$ & $*$ & $*$ & $*$ & $*$ & & & $*$ & $*$ \\
\hline Subprovince: Eastern Mediterranean & $*$ & $*$ & * & * & $*$ & $*$ & $*$ & & & $*$ & * \\
\hline Subprovince: Western Mediterranean & $*$ & $*$ & * & $*$ & $*$ & $*$ & $*$ & & & $*$ & . \\
\hline Subprovince: Caucasian & * & $*$ & & * & * & * & * & & & . & * \\
\hline Province: Macaronesian & & & * & & & & & & & & \\
\hline Province: Irano-Afghan & $*$ & $*$ & $*$ & $*$ & $*$ & * & $*$ & & & $*$ & $*$ \\
\hline Province:Turanian & $*$ & $*$ & & & $*$ & * & $*$ & & & $*$ & $*$ \\
\hline \multicolumn{12}{|l|}{ Province: Asian Montane } \\
\hline Subprovine: Middle Asian & & & & & & & & & & $*$ & \\
\hline Subprovince:Central Asian & $*$ & $*$ & & & & $*$ & $*$ & $*$ & & * & $*$ \\
\hline \multicolumn{12}{|l|}{ Kingdom: Paleotropical } \\
\hline \multicolumn{12}{|l|}{ Subkingdom:Oriental } \\
\hline Province: Taiwanian & & & & & & & & & & & \\
\hline Province: Kashmir-Punjab & * & $*$ & & & & & & & & $*$ & $*$ \\
\hline Province: Sino-Burman & & & & & & & & & & & \\
\hline Province: Indo-Malayan & & & & & & & & & & & \\
\hline Realm: Nearctic & & & & & & & & & & & \\
\hline Kingdom: Neotropical & & & & & & & & & & & \\
\hline
\end{tabular}


Tab. 2 (continuation). Zoogeographical classification of Polish mammalian fauna.

\begin{tabular}{|c|c|c|c|c|c|c|c|c|c|c|c|}
\hline \multirow{2}{*}{$\begin{array}{l}\text { Family } \\
\text { Species }\end{array}$} & \multicolumn{11}{|c|}{ Vespertilionidae } \\
\hline & ENI & ESE & PIP & PPY & PIN & NLA & NYN & NYL & PAR & PAS & BAR \\
\hline No & 23 & 24 & 25 & 26 & 27 & 28 & 29 & 30 & 31 & 32 & 33 \\
\hline \multicolumn{12}{|l|}{ Kingdom: Holarctic } \\
\hline \multicolumn{12}{|l|}{ Realm: Palaearctic } \\
\hline \multicolumn{12}{|l|}{ Region: Western Palaearctic } \\
\hline \multicolumn{12}{|l|}{ Unit: I. Europe } \\
\hline Province: Arctic & * & & & & & & & & & & \\
\hline Province: Western Boreal & * & & * & & * & & * & & * & & * \\
\hline Province: Eastern Boreal & * & & * & & * & & * & & $*$ & & * \\
\hline Province: Atlantic & & * & * & & * & * & * & * & * & * & * \\
\hline Province: Central European & $*$ & * & * & & $*$ & $*$ & $*$ & $*$ & * & $*$ & * \\
\hline Province: Central Russian & * & * & * & & * & * & * & * & * & $*$ & * \\
\hline Province: Submediterranean & $*$ & $*$ & * & & $*$ & $*$ & $*$ & $*$ & $*$ & $*$ & * \\
\hline Province: Western Mediterranean & 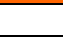 & $*$ & $*$ & & $*$ & $*$ & $*$ & $*$ & $*$ & $*$ & $*$ \\
\hline Province: Eastern Mediterranean & 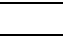 & $*$ & $*$ & & * & $*$ & $*$ & $*$ & $*$ & $*$ & $*$ \\
\hline Province: Saharo-Sind & 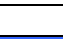 & $*$ & $*$ & & 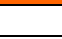 & $*$ & $*$ & $*$ & & $*$ & $*$ \\
\hline Province: Turanian & * & * & * & & 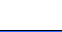 & * & * & 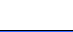 & & 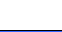 & \\
\hline Province: Iranian & * & * & * & & * & * & * & * & & * & \\
\hline Province: Pontic & & $*$ & $*$ & & * & $*$ & $*$ & $*$ & $*$ & $*$ & $*$ \\
\hline \multicolumn{12}{|l|}{ Region: Eastern Palearctic } \\
\hline Subregion: 1. Arctic & * & & & & & & - & & 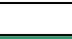 & & \\
\hline Subregion: 2. Boreal-European-Siberian & * & $*$ & & & $*$ & & $*$ & & $*$ & & \\
\hline Province: European-West Siberian & * & $*$ & - & & * & 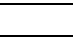 & * & - & - & 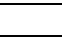 & . \\
\hline Subprovince: Central European & * & * & * & & * & * & * & * & $*$ & * & * \\
\hline Subprovince: Eastern European & $*$ & * & $*$ & & * & $*$ & * & * & $*$ & * & $*$ \\
\hline Subprovince: Western Siberian & $*$ & $*$ & $*$ & & * & & $*$ & & - & & \\
\hline Subprovince: Eastern Siberian & * & $*$ & & & & & & & $*$ & $*$ & \\
\hline Subprovince: Central Siberian & * & $*$ & & & & & 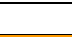 & - & 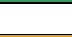 & $*$ & \\
\hline Subregion: 3. Himalayan-Manchurian & 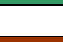 & $*$ & & & & & $*$ & $*$ & $*$ & * & \\
\hline Province: Manchurian-Korean & $*$ & $*$ & & & & & 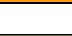 & & 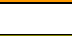 & 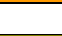 & \\
\hline Province: Japanese & * & & & & & & $*$ & & $*$ & * & \\
\hline Subregion: 4. Mediterranean-Asian & & & * & & * & * & $*$ & * & * & * & \\
\hline \multicolumn{12}{|l|}{ Province: Steppe } \\
\hline Subprovince: Danubian & & $*$ & $*$ & & $*$ & $*$ & $*$ & $*$ & $*$ & $*$ & $*$ \\
\hline Subprovince: Pontic & & $*$ & $*$ & & $*$ & $*$ & $*$ & $*$ & $*$ & $*$ & $*$ \\
\hline Subprovince: Transcarpathian & * & $*$ & $*$ & & $*$ & 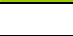 & $*$ & $*$ & $*$ & $*$ & $*$ \\
\hline Subprovince: Ural-Barabin & * & $*$ & $*$ & & * & & & 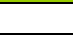 & - & & \\
\hline Subprovince: Mongolian & * & $*$ & & & & & & & $*$ & $*$ & \\
\hline Province: Mediterranean & & $*$ & $*$ & & $*$ & $*$ & $*$ & $*$ & $*$ & $*$ & $*$ \\
\hline Subprovince: Eastern Mediterranean & & * & * & & * & * & * & * & * & * & * \\
\hline Subprovince: Western Mediterranean & & $*$ & $*$ & & $*$ & $*$ & $*$ & $*$ & $*$ & $*$ & $*$ \\
\hline Subprovince: Caucasian & $*$ & $*$ & $*$ & & $*$ & $*$ & $*$ & * & $*$ & $*$ & $*$ \\
\hline Province: Macaronesian & & $*$ & 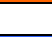 & & 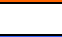 & 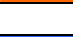 & 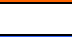 & $*$ & & $*$ & $*$ \\
\hline Province: Irano-Afghan & $*$ & $*$ & $*$ & & $*$ & $*$ & $*$ & $*$ & & $*$ & \\
\hline Province:Turanian & * & $*$ & $*$ & & & $*$ & $*$ & & & & \\
\hline Province: Asian Montane & $*$ & & & & & & $*$ & & & & \\
\hline Subprovine: Middle Asian & * & & * & & & & & & & & \\
\hline Subprovince:Central Asian & * & $*$ & * & & & & & & $*$ & * & \\
\hline Kingdom: Paleotropical & & * & $*$ & & & & $*$ & & & & \\
\hline \multicolumn{12}{|l|}{ Subkingdom:Oriental } \\
\hline \multicolumn{12}{|l|}{ Province: Taiwanian } \\
\hline \multicolumn{12}{|l|}{ Province: Kashmir-Punjab } \\
\hline \multicolumn{12}{|l|}{ Province: Sino-Burman } \\
\hline \multicolumn{12}{|l|}{ Province: Indo-Malayan } \\
\hline \multicolumn{12}{|l|}{ Realm: Nearctic } \\
\hline Kingdom: Neotropical & & & & & & & & & & & \\
\hline
\end{tabular}


Tab. 2 (continuation). Zoogeographical classification of Polish mammalian fauna; * - species whose natural ranges have changed the most significantly over the last few centuries (or decades), ${ }^{(5)}$ - south-western periphery of its range in Poland, ${ }^{(6)}$ - western periphery of its range in Poland.

\begin{tabular}{|c|c|c|c|c|c|c|c|c|c|c|c|}
\hline \multirow{2}{*}{$\begin{array}{rr}\text { Family } \\
\text { Species } \\
\end{array}$} & \multicolumn{3}{|c|}{ Leporidae } & \multicolumn{4}{|c|}{ Sciuridae } & \multirow{2}{*}{\begin{tabular}{|c}
$\begin{array}{c}\text { Casto- } \\
\text { ridae }\end{array}$ \\
CAFIB
\end{tabular}} & \multirow{2}{*}{\begin{tabular}{|c|}
$\begin{array}{c}\text { Crice- } \\
\text { tidae }\end{array}$ \\
CRICRI \\
\end{tabular}} & \multicolumn{2}{|c|}{ Arvicolidae } \\
\hline & $\mathrm{ORCU}$ & LECAP & LETIM & SCVUL & SPECI & SPESU & U/MARMO & & & ONDZ & CLEGL \\
\hline No & $34^{*}$ & $35^{*}$ & $36^{(5)}$ & 37 & $38^{*}$ & $39^{*(6)}$ & 40 & $41 *$ & 42 & $43^{*}$ & 44 \\
\hline \multicolumn{12}{|l|}{ Kingdom: Holarctic } \\
\hline \multicolumn{12}{|l|}{ Realm: Palaearctic } \\
\hline \multicolumn{12}{|l|}{ Region: Western Palaearctic } \\
\hline \multicolumn{12}{|l|}{ Unit: I. Europe } \\
\hline Province: Arctic & & & $*$ & $*$ & & & & $*$ & & $*$ & $*$ \\
\hline Province: Western Boreal & $*$ & $*$ & $*$ & $*$ & & & & $*$ & & $*$ & $*$ \\
\hline Province: Eastern Boreal & & $*$ & $*$ & $*$ & & & & $*$ & & $*$ & $*$ \\
\hline Province: Atlantic & * & * & * & * & & & & * & & $*$ & * \\
\hline Province: Central European & * & * & * & * & * & * & * & * & * & * & * \\
\hline Province: Central Russian & & * & $*$ & * & - & & & $*$ & $*$ & $*$ & $*$ \\
\hline Province: Submediterranean & $*$ & $*$ & & $*$ & $*$ & & $*$ & $*$ & $*$ & $*$ & $*$ \\
\hline Province: Western Mediterranean & $*$ & $*$ & & $*$ & & & & & & & $*$ \\
\hline Province: Eastern Mediterranean & $*$ & $*$ & & $*$ & & & & & - & & $*$ \\
\hline Province: Saharo-Sind & $*$ & $*$ & & & & & & & 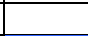 & & \\
\hline Province: Turanian & & $*$ & & & & & & & $*$ & & \\
\hline Province: Iranian & & $*$ & & & $*$ & & & & 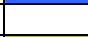 & & \\
\hline Province: Pontic & * & * & & * & * & * & & & * & * & \\
\hline \multicolumn{12}{|l|}{ Region: Eastern Palearctic } \\
\hline Subregion: 1 . Arctic & & 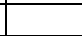 & & $*$ & & & & * & & $*$ & $*$ \\
\hline Subregion: 2. Boreal-European-Siberian & & $*$ & $*$ & $*$ & & & & 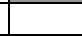 & 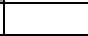 & $*$ & $*$ \\
\hline Province: European-West Siberian & & & $*$ & $*$ & 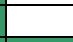 & & & $*$ & $*$ & $*$ & $*$ \\
\hline Subprovince: Central European & $*$ & $*$ & $*$ & $*$ & $*$ & $*$ & $*$ & $*$ & $*$ & $*$ & $*$ \\
\hline Subprovince: Eastern European & $*$ & $*$ & $*$ & $*$ & $*$ & $*$ & $*$ & $*$ & $*$ & $*$ & $*$ \\
\hline Subprovince: Western Siberian & & $*$ & $*$ & $*$ & & & & $*$ & $*$ & $*$ & $*$ \\
\hline Subprovince: Eastern Siberian & & & $*$ & $*$ & & & & $*$ & $*$ & $*$ & \\
\hline Subprovince: Central Siberian & & & $*$ & $*$ & & & & $*$ & $*$ & $*$ & $*$ \\
\hline Subregion: 3. Himalayan-Manchurian & & $*$ & & $*$ & $*$ & & & & & 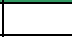 & \\
\hline Province: Manchurian-Korean & & & & * & & & & & & & \\
\hline Province: Japanese & & & $*$ & $*$ & & & & & & & \\
\hline Subregion: 4. Mediterranean-Asian & & $*$ & & $*$ & & & & & & & \\
\hline \multicolumn{12}{|l|}{ Province: Steppe } \\
\hline Subprovince: Danubian & * & * & & * & * & & & & * & * & \\
\hline Subprovince: Pontic & * & $*$ & & $*$ & $*$ & $*$ & & & $*$ & $*$ & \\
\hline Subprovince: Transcarpathian & * & $*$ & & $*$ & & & & & & * & $*$ \\
\hline Subprovince: Ural-Barabin & & $*$ & & $*$ & & & & $*$ & $*$ & $*$ & * \\
\hline Subprovince: Mongolian & & & & $*$ & $*$ & & & $*$ & $*$ & & $*$ \\
\hline Province: Mediterranean & $*$ & $*$ & & $*$ & & & & & & & * \\
\hline Subprovince: Eastern Mediterranean & $*$ & $*$ & & $*$ & & & & & & & $*$ \\
\hline Subprovince: Western Mediterranean & $*$ & $*$ & & $*$ & & & & & & & $*$ \\
\hline Subprovince: Caucasian & & $*$ & & $*$ & $*$ & & & & & & \\
\hline Province: Macaronesian & $*$ & & & & & & & & & & \\
\hline Province: Irano-Afghan & & $*$ & & & * & & & & * & & \\
\hline Province:Turanian & & $*$ & & & & & & & * & & \\
\hline Province: Asian Montane & & & & & & & & & $*$ & & \\
\hline Subprovine: Middle Asian & & & $*$ & $*$ & & & & & & & \\
\hline Subprovince:Central Asian & & $*$ & $*$ & $*$ & & & & $*$ & $*$ & & $*$ \\
\hline \multicolumn{12}{|l|}{ Kingdom: Paleotropical } \\
\hline \multicolumn{12}{|l|}{ Subkingdom:Oriental } \\
\hline \multicolumn{12}{|l|}{ Province: Taiwanian } \\
\hline Province: Kashmir-Punjab & & & & & & & & & & & \\
\hline Province: Sino-Burman & & & & & & & & & & & \\
\hline Province: Indo-Malayan & & & & & & & & & & & \\
\hline Realm: Nearctic & * & * & * & & & & & & & $*$ & \\
\hline Kingdom: Neotropical & * & $*$ & & & & & & & & & \\
\hline
\end{tabular}


Tab. 2 (continuation). Zoogeographical classification of Polish mammalian fauna; * - species whose natural ranges have changed the most significantly over the last few centuries (or decades).

\begin{tabular}{|c|c|c|c|c|c|c|c|c|c|c|c|}
\hline \multirow{2}{*}{\begin{tabular}{c|} 
Family \\
Species
\end{tabular}} & \multicolumn{7}{|c|}{ Arvicolidae } & \multicolumn{4}{|c|}{ Muridae } \\
\hline & ARTER I & PITAT & PITSU & MINIV & MIECO & MIAGR & MIARV & MUSMU & RATNO & RARAT & MIMIN \\
\hline No & 45 & 46 & 47 & 48 & 49 & 50 & 51 & $52 *$ & $53^{*}$ & $54 *$ & 55 \\
\hline \multicolumn{12}{|l|}{ Kingdom: Holarctic } \\
\hline \multicolumn{12}{|l|}{ Realm: Palaearctic } \\
\hline \multicolumn{12}{|l|}{ Region: Western Palaearctic } \\
\hline \multicolumn{12}{|l|}{ Unit: I. Europe } \\
\hline Province: Arctic & $*$ & & & & $*$ & $*$ & & $*$ & $*$ & & \\
\hline Province: Western Boreal & $*$ & & & & $*$ & $*$ & & $*$ & $*$ & $*$ & $*$ \\
\hline Province: Eastern Boreal & $*$ & & & & $*$ & $*$ & & $*$ & $*$ & $*$ & $*$ \\
\hline Province: Atlantic & * & & $*$ & $*$ & $*$ & $*$ & $*$ & $*$ & $*$ & $*$ & $*$ \\
\hline Province: Central European & * & * & $*$ & * & $*$ & $*$ & $*$ & * & * & * & $*$ \\
\hline Province: Central Russian & $*$ & & $*$ & 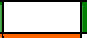 & $*$ & $*$ & $*$ & $*$ & $*$ & $*$ & $*$ \\
\hline Province: Submediterranean & $*$ & & $*$ & $*$ & & $*$ & * & $*$ & $*$ & $*$ & * \\
\hline Province: Western Mediterranean & $*$ & & & $*$ & & $*$ & & $*$ & $*$ & $*$ & \\
\hline Province: Eastern Mediterranean & $*$ & & & $*$ & & & 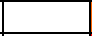 & $*$ & $*$ & $*$ & \\
\hline Province: Saharo-Sind & 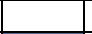 & & & & & & & $*$ & $*$ & $*$ & \\
\hline Province: Turanian & $*$ & & & & & $*$ & $*$ & $*$ & $*$ & $*$ & $*$ \\
\hline Province: Iranian & $*$ & & & $*$ & & & 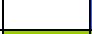 & $*$ & $*$ & $*$ & \\
\hline Province: Pontic & $*$ & & $*$ & & & & $*$ & $*$ & $*$ & $*$ & $*$ \\
\hline \multicolumn{12}{|l|}{ Region: Eastern Palearctic } \\
\hline Subregion: 1. Arctic & * & & & & $*$ & $*$ & & $*$ & $*$ & & \\
\hline Subregion: 2. Boreal-European-Siberian & * & & & & * & $*$ & 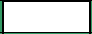 & * & * & * & * \\
\hline Province: European-West Siberian & * & & 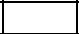 & - & $*$ & $*$ & $*$ & $*$ & $*$ & * & $*$ \\
\hline Subprovince: Central European & $*$ & * & $*$ & * & $*$ & $*$ & $*$ & $*$ & $*$ & $*$ & $*$ \\
\hline Subprovince: Eastern European & $*$ & & $*$ & $*$ & $*$ & $*$ & $*$ & $*$ & $*$ & $*$ & $*$ \\
\hline Subprovince: Western Siberian & $*$ & & & & $*$ & $*$ & $*$ & $*$ & $*$ & $*$ & $*$ \\
\hline Subprovince: Eastern Siberian & $*$ & & & & $*$ & - & & $*$ & $*$ & $*$ & $*$ \\
\hline Subprovince: Central Siberian & * & & & & $*$ & $*$ & & * & $*$ & $*$ & $*$ \\
\hline Subregion: 3. Himalayan-Manchurian & & & & & $*$ & $*$ & $*$ & $*$ & $*$ & $*$ & $*$ \\
\hline Province: Manchurian-Korean & & & & & & & & $*$ & * & $*$ & $*$ \\
\hline Province: Japanese & & & & & & & & * & * & $*$ & * \\
\hline Subregion: 4. Mediterranean-Asian & * & & & & & & & $*$ & $*$ & $*$ & \\
\hline \multicolumn{12}{|l|}{ Province: Steppe } \\
\hline Subprovince: Danubian & * & & $*$ & & & & * & $*$ & $*$ & $*$ & $*$ \\
\hline Subprovince: Pontic & * & & $*$ & & & & $*$ & $*$ & $*$ & $*$ & $*$ \\
\hline Subprovince: Transcarpathian & $*$ & $*$ & $*$ & $*$ & & $*$ & $*$ & $*$ & $*$ & $*$ & $*$ \\
\hline Subprovince: Ural-Barabin & & & & & & & $*$ & $*$ & $*$ & $*$ & $*$ \\
\hline Subprovince: Mongolian & * & & & & $*$ & $*$ & * & $*$ & * & $*$ & \\
\hline Province: Mediterranean & $*$ & & & $*$ & & $*$ & & $*$ & $*$ & $*$ & \\
\hline Subprovince: Eastern Mediterranean & $*$ & & & $*$ & & & & $*$ & $*$ & $*$ & \\
\hline Subprovince: Western Mediterranean & $*$ & & & $*$ & & $*$ & & $*$ & $*$ & $*$ & \\
\hline Subprovince: Caucasian & $*$ & & & $*$ & & & $*$ & * & $*$ & $*$ & $*$ \\
\hline Province: Macaronesian & & & & & & & & & $*$ & $*$ & \\
\hline Province: Irano-Afghan & $*$ & & & $*$ & & & & * & $*$ & $*$ & \\
\hline Province:Turanian & $*$ & & & & & $*$ & $*$ & $*$ & $*$ & $*$ & $*$ \\
\hline Province: Asian Montane & & & & & & & & * & $*$ & $*$ & \\
\hline Subprovine: Middle Asian & & & & & & & & $*$ & $*$ & $*$ & 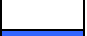 \\
\hline Subprovince:Central Asian & * & & & & $*$ & $*$ & $*$ & $*$ & $*$ & $*$ & $*$ \\
\hline Kingdom: Paleotropical & & & & & & & & * & * & * & $*$ \\
\hline Subkingdom:Oriental & & & & & & & & * & * & * & $*$ \\
\hline Province: Taiwanian & & & & & & & & * & * & * & $*$ \\
\hline Province: Kashmir-Punjab & & & & & & & & * & * & * & $*$ \\
\hline Province: Sino-Burman & & & & & & & & * & * & * & $*$ \\
\hline Province: Indo-Malayan & & & & & & & & $*$ & $*$ & $*$ & $*$ \\
\hline Realm: Nearctic & & & & & $*$ & & & $*$ & * & $*$ & \\
\hline Kingdom: Neotropical & & & & & & & & $*$ & * & $*$ & \\
\hline
\end{tabular}


Tab. 2 (continuation). Zoogeographical classification of Polish mammalian fauna; * - species whose natural ranges have changed the most significantly over the last few centuries (or decades), ${ }^{(7)}$ - north-western border of the range in Poland.

\begin{tabular}{|c|c|c|c|c|c|c|c|c|c|c|c|}
\hline \multirow{2}{*}{$\begin{array}{r}\text { Family } \\
\text { Species }\end{array}$} & \multicolumn{4}{|c|}{ Muridae } & \multicolumn{2}{|c|}{ Zapodidae } & \multicolumn{4}{|c|}{ Gliridae } & \multirow{2}{*}{$\begin{array}{l}\text { Ursidae } \\
\text { URSAR }\end{array}$} \\
\hline & APAGR & APMIC & APSYL & APFLA & SIBET & SISUB & ELQUE & DRNIT & GLIGLI & MUAVE & \\
\hline No & 56 & 57 & 58 & $59 *$ & 60 & 61 & 62 & $63^{(7)}$ & 64 & 65 & 66 \\
\hline \multicolumn{12}{|l|}{ Kingdom: Holarctic } \\
\hline \multicolumn{12}{|l|}{ Realm: Palaearctic } \\
\hline \multicolumn{12}{|l|}{ Region: Western Palaearctic } \\
\hline \multicolumn{12}{|l|}{ Unit: I. Europe } \\
\hline Province: Arctic & & & $*$ & & & & & & & & $*$ \\
\hline Province: Western Boreal & & & $*$ & $*$ & $*$ & & & & & & $*$ \\
\hline Province: Eastern Boreal & $*$ & & & $*$ & $*$ & & & & & & $*$ \\
\hline Province: Atlantic & & & $*$ & $*$ & & & $*$ & & * & * & $*$ \\
\hline Province: Central European & $*$ & * & $*$ & * & $*$ & & * & * & $*$ & * & $*$ \\
\hline Province: Central Russian & * & 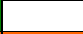 & * & * & * & & * & * & * & * & * \\
\hline Province: Submediterranean & $*$ & $*$ & $*$ & $*$ & & & $*$ & $*$ & $*$ & $*$ & $*$ \\
\hline Province: Western Mediterranean & & & $*$ & $*$ & & & $*$ & $*$ & * & $*$ & $*$ \\
\hline Province: Eastern Mediterranean & $*$ & & $*$ & * & & & $*$ & $*$ & $*$ & * & $*$ \\
\hline Province: Saharo-Sind & & & $*$ & & & & $*$ & 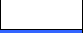 & & & \\
\hline Province: Turanian & $*$ & & $*$ & & * & & & $*$ & & & \\
\hline Province: Iranian & & & $*$ & & & & & $*$ & $*$ & & $*$ \\
\hline Province: Pontic & $*$ & $*$ & $*$ & $*$ & & & & & & & \\
\hline \multicolumn{12}{|l|}{ Region: Eastern Palearctic } \\
\hline Subregion: 1. Arctic & & & $*$ & & & & & & & & $*$ \\
\hline Subregion: 2. Boreal-European-Siberian & $*$ & & $*$ & & $*$ & & & 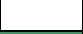 & & & $*$ \\
\hline Province: European-West Siberian & $*$ & 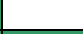 & $*$ & $*$ & $*$ & & . & $*$ & & & $*$ \\
\hline Subprovince: Central European & $*$ & $*$ & $*$ & $*$ & $*$ & & $*$ & $*$ & $*$ & $*$ & $*$ \\
\hline Subprovince: Eastern European & $*$ & $*$ & $*$ & $*$ & $*$ & & $*$ & $*$ & $*$ & $*$ & $*$ \\
\hline Subprovince: Western Siberian & $*$ & & $*$ & $*$ & $*$ & & $*$ & $*$ & $*$ & & $*$ \\
\hline Subprovince: Eastern Siberian & $*$ & & $*$ & * & $*$ & & & & & & $*$ \\
\hline Subprovince: Central Siberian & $*$ & & $*$ & $*$ & $*$ & & & & & & $*$ \\
\hline Subregion: 3. Himalayan-Manchurian & $*$ & & $*$ & $*$ & & & & & & & $*$ \\
\hline Province: Manchurian-Korean & $*$ & & & * & & & & & & & $*$ \\
\hline Province: Japanese & $*$ & & & & & & & & & & $*$ \\
\hline Subregion: 4. Mediterranean-Asian & & & $*$ & & & & & $*$ & & & $*$ \\
\hline \multicolumn{12}{|l|}{ Province: Steppe } \\
\hline Subprovince: Danubian & $*$ & $*$ & $*$ & $*$ & & & & & & & \\
\hline Subprovince: Pontic & $*$ & $*$ & $*$ & $*$ & & & & & & & \\
\hline Subprovince: Transcarpathian & $*$ & $*$ & $*$ & * & $*$ & & * & * & * & * & $*$ \\
\hline Subprovince: Ural-Barabin & $*$ & & $*$ & & $*$ & & $*$ & & & & $*$ \\
\hline Subprovince: Mongolian & $*$ & & & & & & & & & & $*$ \\
\hline Province: Mediterranean & & & $*$ & $*$ & & & $*$ & $*$ & $*$ & $*$ & $*$ \\
\hline Subprovince: Eastern Mediterranean & $*$ & & $*$ & $*$ & & & $*$ & * & $*$ & $*$ & $*$ \\
\hline $\begin{array}{l}\text { Subprovince: Western } \\
\text { Mediterranean }\end{array}$ & & & $*$ & $*$ & & & $*$ & $*$ & $*$ & * & $*$ \\
\hline Subprovince: Caucasian & $*$ & & $*$ & $*$ & $*$ & & & $*$ & $*$ & & $*$ \\
\hline \multicolumn{12}{|l|}{ Province: Macaronesian } \\
\hline Province: Irano-Afghan & & & $*$ & & & & & $*$ & $*$ & & $*$ \\
\hline Province:Turanian & $*$ & & $*$ & & $*$ & & & $*$ & & & \\
\hline Province: Asian Montane & & & & & & & & * & & & $*$ \\
\hline Subprovine: Middle Asian & & & $*$ & & $*$ & & & $*$ & & & $*$ \\
\hline Subprovince:Central Asian & $*$ & & $*$ & & $*$ & & & $*$ & & & $*$ \\
\hline Kingdom: Paleotropical & $*$ & & & $*$ & & & & & & & \\
\hline Subkingdom:Oriental & $*$ & & & & & & & & & & \\
\hline Province: Taiwanian & $*$ & & & & & & & & & & \\
\hline \multicolumn{12}{|l|}{ Province: Kashmir-Punjab } \\
\hline \multicolumn{12}{|l|}{ Province: Sino-Burman } \\
\hline \multicolumn{12}{|l|}{ Province: Indo-Malayan } \\
\hline Realm: Nearctic & & & & & & & & & & & * \\
\hline Kingdom: Neotropical & & & & & & & & & & & \\
\hline
\end{tabular}


Tab. 2 (continuation). Zoogeographical classification of Polish mammalian fauna; * - species whose natural ranges have changed the most significantly over the last few centuries (or decades).

\begin{tabular}{|c|c|c|c|c|c|c|c|c|c|c|c|}
\hline Family & & Canidae & & & & & Mustelida & & & & \\
\hline Species & CALUP & VUVU & NYPRO & MEMEL & LUTLU & MAMAR & MARFO & MUPU & MUEV & MUER & MUNI \\
\hline No & 67 & 68 & 69 & 70 & 71 & 72 & 73 & $74 *$ & 75 & $76^{*}$ & $77 *$ \\
\hline \multicolumn{12}{|l|}{ Kingdom: Holarctic } \\
\hline \multicolumn{12}{|l|}{ Realm: Palaearctic } \\
\hline \multicolumn{12}{|l|}{ Region: Western Palaearctic } \\
\hline \multicolumn{12}{|l|}{ Unit: I. Europe } \\
\hline Province: Arctic & $*$ & $*$ & & & $*$ & $*$ & & & & $*$ & $*$ \\
\hline Province: Western Boreal & $*$ & $*$ & & $*$ & $*$ & $*$ & & $*$ & & $*$ & $*$ \\
\hline Province: Eastern Boreal & $*$ & $*$ & $*$ & $*$ & $*$ & $*$ & & $*$ & & $*$ & $*$ \\
\hline Province: Atlantic & $*$ & $*$ & & $*$ & $*$ & $*$ & $*$ & $*$ & & $*$ & $*$ \\
\hline Province: Central European & * & $*$ & * & $*$ & $*$ & $*$ & $*$ & $*$ & * & * & * \\
\hline Province: Central Russian & $*$ & $*$ & $*$ & $*$ & $*$ & $*$ & 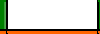 & $*$ & 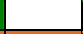 & $*$ & * \\
\hline Province: Submediterranean & $*$ & $*$ & & $*$ & $*$ & $*$ & $*$ & $*$ & $*$ & $*$ & $*$ \\
\hline Province: Western Mediterranean & $*$ & $*$ & & $*$ & $*$ & $*$ & $*$ & $*$ & & & $*$ \\
\hline Province: Eastern Mediterranean & * & $*$ & & $*$ & $*$ & $*$ & * & $*$ & & & $*$ \\
\hline Province: Saharo-Sind & & $*$ & & 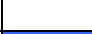 & $*$ & & & $*$ & 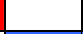 & 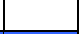 & $*$ \\
\hline Province: Turanian & $*$ & $*$ & & $*$ & & . & $*$ & & $*$ & $*$ & $*$ \\
\hline Province: Iranian & $*$ & . & & * & 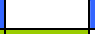 & $*$ & $*$ & 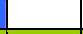 & & 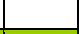 & . \\
\hline Province: Pontic & * & $*$ & * & * & $*$ & & * & $*$ & * & $*$ & * \\
\hline \multicolumn{12}{|l|}{ Region: Eastern Palearctic } \\
\hline Subregion: 1. Arctic & $*$ & $*$ & . & 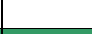 & $*$ & $*$ & & & & $*$ & $*$ \\
\hline Subregion: 2. Boreal-European-Siberian & $*$ & $*$ & * & $*$ & $*$ & $*$ & & & & $*$ & * \\
\hline Province: European-West Siberian & $*$ & $*$ & $*$ & $*$ & $*$ & $*$ & & & & $*$ & $*$ \\
\hline Subprovince: Central European & $*$ & $*$ & $*$ & $*$ & $*$ & $*$ & $*$ & $*$ & * & $*$ & $*$ \\
\hline Subprovince: Eastern European & $*$ & $*$ & $*$ & $*$ & $*$ & $*$ & $*$ & $*$ & $*$ & $*$ & $*$ \\
\hline Subprovince: Western Siberian & $*$ & $*$ & $*$ & $*$ & $*$ & $*$ & & & 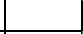 & $*$ & $*$ \\
\hline Subprovince: Eastern Siberian & $*$ & $*$ & * & $*$ & $*$ & & & & 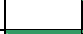 & $*$ & * \\
\hline Subprovince: Central Siberian & $*$ & * & * & * & * & & & & * & $*$ & * \\
\hline Subregion: 3. Himalayan-Manchurian & $*$ & $*$ & $*$ & $*$ & $*$ & & $*$ & & $*$ & $*$ & 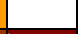 \\
\hline Province: Manchurian-Korean & * & $*$ & $*$ & * & & & & & & $*$ & * \\
\hline Province: Japanese & * & $*$ & $*$ & $*$ & $*$ & & & & & * & $*$ \\
\hline Subregion: 4. Mediterranean-Asian & $*$ & $*$ & & $*$ & . & $*$ & $*$ & & & 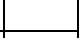 & $*$ \\
\hline \multicolumn{12}{|l|}{ Province: Steppe } \\
\hline Subprovince: Danubian & & $*$ & $*$ & $*$ & $*$ & $*$ & $*$ & $*$ & $*$ & $*$ & $*$ \\
\hline Subprovince: Pontic & $*$ & $*$ & $*$ & $*$ & $*$ & 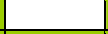 & $*$ & $*$ & $*$ & $*$ & $*$ \\
\hline Subprovince: Transcarpathian & $*$ & $*$ & $*$ & $*$ & $*$ & $*$ & $*$ & $*$ & 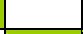 & $*$ & * \\
\hline Subprovince: Ural-Barabin & $*$ & $*$ & & $*$ & $*$ & & & & * & $*$ & * \\
\hline Subprovince: Mongolian & $*$ & $*$ & & $*$ & & & $*$ & & $*$ & & $*$ \\
\hline Province: Mediterranean & $*$ & $*$ & & $*$ & $*$ & $*$ & $*$ & $*$ & & & $*$ \\
\hline Subprovince: Eastern Mediterranean & $*$ & $*$ & & $*$ & $*$ & $*$ & $*$ & $*$ & & & $*$ \\
\hline $\begin{array}{l}\text { Subprovince: Western } \\
\text { Mediterranean }\end{array}$ & * & $*$ & & $*$ & $*$ & $*$ & $*$ & $*$ & & & * \\
\hline Subprovince: Caucasian & $*$ & $*$ & * & $*$ & $*$ & $*$ & $*$ & & & $*$ & * \\
\hline Province: Macaronesian & & & & & & & & & & & * \\
\hline Province: Irano-Afghan & $*$ & & & $*$ & & $*$ & $*$ & & & & \\
\hline Province:Turanian & $*$ & $*$ & & * & & & $*$ & & $*$ & $*$ & $*$ \\
\hline Province: Asian Montane & $*$ & & & & & & $*$ & & & & \\
\hline Subprovine: Middle Asian & $*$ & & & & & & $*$ & & $*$ & & \\
\hline Subprovince:Central Asian & $*$ & $*$ & & $*$ & & & $*$ & & $*$ & $*$ & $*$ \\
\hline Kingdom: Paleotropical & $*$ & $*$ & & & $*$ & & & & & & \\
\hline Subkingdom:Oriental & & & & $*$ & $*$ & & & & & & \\
\hline \multicolumn{12}{|l|}{ Province: Taiwanian } \\
\hline Province: Kashmir-Punjab & $*$ & $*$ & & & & & & & & & \\
\hline Province: Sino-Burman & $*$ & $*$ & & & & & & & & & \\
\hline \multicolumn{12}{|l|}{ Province: Indo-Malayan } \\
\hline Realm: Nearctic & $*$ & $*$ & & & & & & & & $*$ & $*$ \\
\hline Kingdom: Neotropical & & & & & & & & & & & \\
\hline
\end{tabular}


Tab. 2 (continuation). Zoogeographical classification of Polish mammalian fauna; * - species whose natural ranges have changed the most significantly over the last few centuries (or decades), ${ }^{(8)}$ - northernmost enclave in Poland.

\begin{tabular}{|c|c|c|c|c|c|c|c|c|c|c|c|c|}
\hline Family & Mus- & Felic & dae & Suidae & & & Cervida & & & & Bovidae & \\
\hline Species & MUVIS & FELYN & FESIL & SUSC & ALAL & CACA & CEREL & CERNI & DADA & BISBO & RURUP & OVAM \\
\hline No & $78 *$ & 79 & $80 *$ & $81 *$ & $82 *$ & $83 *$ & $84 *$ & $85 *$ & $86^{*}$ & $87 *$ & $88^{*(8)}$ & 89 \\
\hline Kingdom: Holarctic & & & & & & & & & & & & \\
\hline Realm: Palaearctic & & & & & & & & & & & & \\
\hline Region: Western Palaearctic & & & & & & & & & & & & \\
\hline Unit: I. Europe & & & & & & & & & & & & \\
\hline Province: Arctic & $*$ & * & & & $*$ & $*$ & $*$ & & & & & \\
\hline Province: Western Boreal & $*$ & * & & & $*$ & $*$ & $*$ & & & & & \\
\hline Province: Eastern Boreal & $*$ & $*$ & & & $*$ & $*$ & $*$ & & & & & \\
\hline Province: Atlantic & $*$ & $*$ & $*$ & $*$ & & $*$ & $*$ & $*$ & * & & & $*$ \\
\hline Province: Central European & $*$ & $*$ & $*$ & * & $*$ & * & $*$ & * & * & $*$ & $*$ & * \\
\hline Province: Central Russian & * & * & * & * & $*$ & * & * & * & & & & \\
\hline Province: Submediterranean & $*$ & $*$ & $*$ & $*$ & & $*$ & $*$ & $*$ & $*$ & & $*$ & $*$ \\
\hline Province: Western Mediterranean & $*$ & $*$ & $*$ & $*$ & & $*$ & $*$ & * & * & & & $*$ \\
\hline Province: Eastern Mediterranean & & $*$ & $*$ & $*$ & & $*$ & $*$ & & * & & & $*$ \\
\hline Province: Saharo-Sind & 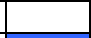 & & $*$ & $*$ & & $*$ & $*$ & & $*$ & & & 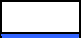 \\
\hline Province: Turanian & $*$ & & & $*$ & & 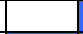 & $*$ & & 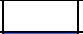 & & & $*$ \\
\hline Province: Iranian & 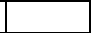 & & $*$ & $*$ & & $*$ & $*$ & & * & & & * \\
\hline Province: Pontic & $*$ & & & $*$ & & & $*$ & & & & & \\
\hline Region: Eastern Palearctic & & & & & & & & & & & & \\
\hline Subregion: 1. Arctic & * & * & & 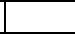 & * & $*$ & * & & & & & \\
\hline Subregion: 2. Boreal-European-Siberian & $*$ & $*$ & & 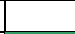 & $*$ & $*$ & $*$ & & & & & \\
\hline Province: European-West Siberian & $*$ & * & & * & $*$ & $*$ & $*$ & $*$ & & & & \\
\hline Subprovince: Central European & $*$ & $*$ & $*$ & $*$ & $*$ & $*$ & $*$ & * & * & $*$ & $*$ & * \\
\hline Subprovince: Eastern European & $*$ & $*$ & $*$ & $*$ & $*$ & $*$ & $*$ & $*$ & * & $*$ & $*$ & $*$ \\
\hline Subprovince: Western Siberian & $*$ & * & & * & $*$ & $*$ & $*$ & * & & & & \\
\hline Subprovince: Eastern Siberian & $*$ & $*$ & & $*$ & $*$ & $*$ & $*$ & * & & & & \\
\hline Subprovince: Central Siberian & $*$ & * & & * & $*$ & $*$ & $*$ & * & & & & \\
\hline Subregion: 3. Himalayan-Manchurian & & $*$ & $*$ & & $*$ & $*$ & $*$ & $*$ & & & & $*$ \\
\hline Province: Manchurian-Korean & $*$ & & & 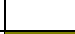 & & $*$ & & $*$ & & & & \\
\hline Province: Japanese & $*$ & & & * & & & & * & & & & \\
\hline Subregion: 4. Mediterranean-Asian & & & $*$ & & & $*$ & $*$ & & * & & & $*$ \\
\hline Province: Steppe & & & & & & & & & & & & \\
\hline Subprovince: Danubian & & & & $*$ & & & & & $*$ & & & \\
\hline Subprovince: Pontic & * & & & * & & & $*$ & & & & & \\
\hline Subprovince: Transcarpathian & & * & $*$ & * & & * & $*$ & & * & & $*$ & $*$ \\
\hline Subprovince: Ural-Barabin & * & & & * & $*$ & $*$ & * & & & & & \\
\hline Subprovince: Mongolian & & $*$ & $*$ & $*$ & $*$ & $*$ & & & & & & $*$ \\
\hline Province: Mediterranean & $*$ & & $*$ & $*$ & & $*$ & $*$ & & * & & & * \\
\hline Subprovince: Eastern Mediterranean & & $*$ & $*$ & $*$ & & $*$ & $*$ & & $*$ & & & $*$ \\
\hline Subprovince: Western Mediterranean & $*$ & $*$ & $*$ & $*$ & & $*$ & $*$ & $*$ & * & & & $*$ \\
\hline Subprovince: Caucasian & $*$ & * & $*$ & $*$ & & $*$ & $*$ & $*$ & & * & $*$ & * \\
\hline Province: Macaronesian & & & & & & & & & & & & $*$ \\
\hline Province: Irano-Afghan & & & * & $*$ & & * & * & & * & & & $*$ \\
\hline Province:Turanian & * & & & $*$ & & & * & & & & & $*$ \\
\hline Province: Asian Montane & & & & & & $*$ & * & & & & * & $*$ \\
\hline Subprovine: Middle Asian & & * & * & & & $*$ & $*$ & & & & * & $*$ \\
\hline Subprovince:Central Asian & $*$ & $*$ & $*$ & & $*$ & $*$ & $*$ & & & & & $*$ \\
\hline Kingdom: Paleotropical & & & $*$ & $*$ & & & & & & & & \\
\hline Subkingdom:Oriental & & & & $*$ & & & * & * & & & & * \\
\hline Province: Taiwanian & & & & 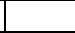 & & & & $*$ & & & & \\
\hline Province: Kashmir-Punjab & & & $*$ & $*$ & & & $*$ & & & & & $*$ \\
\hline Province: Sino-Burman & & & $*$ & $*$ & & & $*$ & & & & & $*$ \\
\hline Province: Indo-Malayan & & & & $*$ & & & $*$ & $*$ & & & & \\
\hline Realm: Nearctic & * & & & $*$ & * & $*$ & $*$ & $*$ & * & & & $*$ \\
\hline Kingdom: Neotropical & & & & & & $*$ & $*$ & & * & & & $*$ \\
\hline
\end{tabular}


Table 2 (explanations). Acronyms of Polish mammal species used in the Table:

Family: Erinaceidae

1. Western hedgehog (Erinaceus europaeus)

2. Eastern hedgehog (Erinaceus concolor) Family: Talpidae

3. Common mole (Talpa europaea) Family: Soricidae

4. Common shrew (Sorex araneus)

5. Masked shrew (Sorex caecutiens)

6. Pygmy shrew (Sorex minutus)

7. Alpine shrew (Sorex alpinus)

8. Water shrew (Neomys fodiens)

9. Miller's water shrew (Neomys anomalus)

10. White-toothed shrew (Crocidura leucodon)

11. Lesser shrew (Crocidura suaveolens) Family: Rhinolophidae

12. Lesser horseshoe bat (Rhinolphus hipposideros) RHH

12. Lesser horseshoe bat (Rhinolphus hipposideros) RHH
13. Greater horseshoe bat (Rhinolophus ferrumequinum) RHF Family: Vespertilionidae

14. Greatr mouse-eared bat (Myotis myotis)

15. Bechstein's bat (Myotis bechsteinii)

16. Natterer's bat (Myotis nattereri)

17. Geoffroy's bat (Myotis emarginatus)

18. Whiskered bat (Myotis mystacinus)

19. Brandt's bat (Myotis brandtii)

20. Pond bat (Myotis dasycneme)

21. Daubenton's bat (Myotis daubentonii)

22. Parti-coloured bat (Vespertilio murinus)

23. Northern bat (Eptesicus nilssonii)

24. Serotine (Eptesicus serotinus)

25. Common pipistrelle (Pipistrellus pipistrellus)

26. Soprano pipistrelle (Pipistrellus pygmaeus)

27. Nathusius' pipistrelle (Pipistrellus nathusii)

28. Greater noctule (Nyctalus lasiopterus)

29. Noctule (Nyctalus noctula)

30. Leisler's bat (Nyctalus leisleri)

31. Brown long-eared bat (Plecotus auritus)

32. Grey long-eared bat (Plecotus austriacus)

33. Barbastelle (Barbastella barbastellus) Family: Leporidae

34. Rabbit (Oryctolagus cuniculus)

35. Cape hare (Lepus capensis)

36. Mountain hare (Lepus timidus) Family: Sciuridae

37. Red squirrel (Sciurus vulgaris)

38. European souslik (Spermophilus citellus)

39. Spotted souslik (Spermophilus suslicus)

40. Alpine marmot (Marmota marmota) Family: Castoridae

41. Eurasian beaver (Castor fiber) Family: Cricetidae

42. Common hamster (Cricetus cricetus) Family: Arvicolidae

43. Muskrat (Ondatra zibethicus)

44. Bank vole (Clethrionomys glareolus)

45. Water vole (Arvicola terrestris)

46. Tatra pine vole (Pitymys tatricus)

47. Common pine vole (Pitymys subterraneus)

48. Snow vole (Microtus nivalis)

49. Root vole (Microtus oeconomus)

50. Field vole (Microtus agrestis)

51. Field mouse (Microtus arvalis)
EREU

ERCON

TALEU

SOARA

SOCAE

SOMIN

SOALP

NEFOD

NEANO

CROLE

CRSUA

MYM

MBE

MYN

MEM

MYS

MYB

MDS

MDA

VMU

ENI

ESE

PIP

PPY

PIN

NLA

NYN

NYL

PAR

PAS

BAR

ORCU

LECAP

LETIM

SCVUL

SPECI

SPESU

MARMO

CAFIB

CRICRI

ONDZI

CLEGL

ARTER

PITAT

PITSU

MINIV

MIECO

MIAGR

MIARV
Family: Muridae

52. Eastern house mouse (Mus musculus) MUSMU

53. Brown rat (Rattus norvegicus)

RATNO

54. Black rat (Rattus rattus)

55. Harvest mouse (Micromys minutus)

RARAT

56. Striped field mouse (Apodemus agrarius)

MIMIN

57. Pygmy field mouse (Apodemus microps)

APAGR

APMIC

APSYL

59. Yellow-necked mouse (Apodemus flavicollis) APFLA Family: Zapodidae

60. Northern birch mouse (Sicista betulina) SIBET

61. Southern birch mouse (Sicista subtilis) SISUB Family: Gliridae

62. Garden dormouse (Eliomys quercinus) ELQUE

63. Forest dormouse (Dryomys nitedula) DRNIT

64. Edible dormouse (Glis glis) GLIGLI

65. Common dormouse (Muscardinus avellanarius) MUAVE Family: Ursidae

66. Brown bear (Ursus arctos) Family: Canidae

67. Wolf (Canis lupus)

URSAR

68. Red fox (Vulpes vulpes)

CALUP

69. Raccoon dog (Nyctereutes procyonoides)

VUVU Family: Mustelidae

70. Badger (Meles meles)

NYPRO

71. Otter (Lutra lutra)

72. Pine marten (Martes martes)

73. Beech marten, stone marten (Martes foina)

74. Western polecat (Mustela putorius)

75. Steppe polecat (Mustela eversmanni)

76. Stoat (Mustela erminea)

77. Weasel (Mustela nivalis)

78. American mink (Mustela vison)

Family: Felidae

79. Lynx (Felis lynx)

80. Wild cat (Felis silvestris)

Family: Suidae

81. Wild boar (Sus scrofa) Family: Cervidae

82. Elk, moose (Alces alces)

MEMEL

LUTLU

MAMAR

MARFO

MUPU

MUEV

MUER

MUNI

MUVIS

FELYN

FESIL

SUSC

ALAL

CACA

CEREL

CERNI

85. Sika deer (Cervus nippon)

86. Fallow deer (Dama dama)

DADA

Family: Bovidae

87. European bison (Bison bonasus)

BISBO

88. Southern chamois (Rupicapra rupicapra)

RURUP

89. Mouflon (Ovis ammon)
OVAM 
With a degree of approximation, the ranges of 11 species can be regarded as confined to Europe. These are: western hedgehog (Erinaceus europaeus), alpine shrew (Sorex alpinus), Miller's water shrew (Neomys anomalus), barbastelle (Barbastella barbastellus), spotted souslik (Spermophilus suslicus), Alpine marmot (Marmota marmota), Tatra pine vole (Pitymys taricus) (see above), common pine vole (Pitymys subterraneus), pygmy field mouse (Apodemus microps), common dormouse (Muscardinus avellanarius) and European bison (Bison bonasus).

Tab. 3. Species richness of Polish mammals by order and family.

\begin{tabular}{|c|c|}
\hline \multicolumn{1}{|c|}{ Taxon } & Number of species \\
\hline Order: Insectivora & $\mathbf{1 1}$ \\
Erinaceidae (hedgehogs) & 2 \\
Talpidae (moles) & 1 \\
Soricidae (shrews) & $\mathbf{2 2}$ \\
Order: Chiroptera & 2 \\
Rhinolophidae (horseshoe bats) & 20 \\
Vespertilionidae (vespertilionid bats) & $\mathbf{3}$ \\
Order: Lagomorpha & 3 \\
Leporidae (hares and rabbits) & $\mathbf{2 9}$ \\
Order: Rodentia & 4 \\
Sciuridae (squirrels) & 1 \\
Castoridae (beavers) & 1 \\
Cricetidae (hamsters) & 9 \\
Arvicolidae (voles) & 8 \\
Muridae (rats and mice) & 2 \\
Zapodidae (birch mice) & 4 \\
Gliridae (dormice) & $\mathbf{1 5}$ \\
Ursidae (bears) & 1 \\
Canidae (dogs) & 3 \\
Mustelidae (weasels and others) & 9 \\
Felidae (cats) & 2 \\
Order: Carnidae (pigs) & $\mathbf{9}$ \\
Cervidae (deer) & 1 \\
Bovidae (cattle) & 5 \\
Order: Artiodactyla & 3 \\
\hline
\end{tabular}

The ranges of the other species extend considerably beyond Europe and are associated with the whole of Eurasia or a part of this continent. They can also be found on other continents (Table 2).

\section{The ranges of Polish mammals outside the Palaearctic. Secondary ranges. Alien species}

Some Polish mammals can be found far away from the Palaearctic, in the Oriental Subkingdom (Paleotropical Kingdom), in North America (Nearctic Realm $=$ Nearctic Kingdom), South America (Neotropical Kingdom) and even in New Zealand, on the Solomon Islands, in Australia (Australian Kingdom), Oceania, in Tasmania, Fiji, Hawaii and the Kerguelen Islands.

The occurrence of other species, such as the mountain hare (Lepus timidus), in the Arctic is associated with the Alaskan tundra zone as far as Labrador and Greenland.

Some 'Polish' species can be found on the Australian continent (Australian Kingdom) and the Holoantarctic Kingdom, which apart from the Antarctic includes New Zealand, Tasmania and Oceania. These are: the wild boar (Sus scrofa), introduced into Australia, Tasmania and New Zealand as a game animal, which is also found on many of the islands in the area extending from Malacca to the Solomon Islands; the fallow deer (Dama dama), introduced into Australia and on 
some Oceanian islands (New Zealand, Fiji); the sika deer (Cervus nippon), introduced into New Zealand and Australia, the red deer (Cervus elaphus), introduced into Australia and New Zealand; the western hedgehog (Erinaceus europaeus), introduced into New Zealand; as well as accidentally introduced synanthropic rodents: the eastern house mouse (Mus musculus), the black rat (Rattus rattus) and the brown rat (Rattus norvegicus); and the carnivores: stoat (Mustela erminea) and weasel (Mustela nivalis), introduced into New Zealand.

The ranges of 25 species stretch as far as South-East Asia (Oriental Subkingdom) (Table 2 \& 4). They are associated with the Far Eastern Provinces of Taiwan, Kashmir-Punjab, SinoBurman and Indo-Malayan. They include one species of shrew, 9 species of bat, 6 rodent species, 5 carnivore species and 4 species of even-toed ungulates (artiodactylous) (Table 2). Apparently, the ranges of most of them (as many as 18) reach the Kashmir-Punjab Province, while 12 reach the Sino-Burman Province and 8 can be found in Taiwan. The Indo-Malayan Province, which includes the Indian Peninsula, the Indochina Peninsula, the Philippines, the Malayan Archipelago, New Guinea, Melanesia, Micronesia and islands in the Pacific within the tropical belt is the limit of distribution of 9 species: the serotine bat (Eptesicus serotinus), 4 species of rodents, the otter (Lutra lutra), the wild boar (Sus scrofa), the red deer (Cervus elaphus) and the sika deer (Cervus nippon).

Tab. 4. Number of species with ranges extending beyond the Palaearctic

\begin{tabular}{|l|c|}
\hline \multicolumn{1}{|c|}{ Unit of zoogeography } & Number of species \\
\hline Holarctic Kingdom & 21 \\
Nearctic Realm & 25 \\
Paleotropical Kingdom & 9 \\
Oriental Subkingdom & 9 \\
Neotropical Kingdom & \\
\hline
\end{tabular}

North America (Nearctic Kingdom) supports 21 species of mammals whose ranges include the area of Poland. These are: three species of Lagomorpha (rabbit Oryctolagus cuniculus, cape hare Lepus capensis, mountain hare Lepus timidus), 5 species of rodents (including the muskrat Ondatra zibethicus), 6 species of Carnivora (brown bear Ursus arctos, wolf Canis lupus, red fox Vulpes vulpes, stoat Mustela erminea, weasel Mustela nivalis, American mink Mustela vison) and 7 species of even-toed ungulates (artiodactylous), including the roe deer (Capreolus capreolus), elk (Alces alces) and red deer (Cervus elaphus); all of which migrated into North America from north-eastern Asia using a land bridge that existed in the Pleistocoene where the Bering Strait is now. The red deer was later introduced into South America, Australia and New Zealand. The other 'Polish' ungulates in North America are the wild boar (Sus scrofa), sika deer (Cervus nippon), fallow deer (Dama dama) and mouflon (Ovis ammon) (Komosińska \& Podsiadło 2002, Goszczyński 1995, Goszczyński et al. 1994, Andrzejewski \& Pielowski 2003, Wilson \& Reeder 1993).

Ten 'Polish' mammal species are also distributed in South America (Neotropical Kingdom). This Kingdom consists of the southernmost parts of Florida, the Pacific shores of Mexico, Central America, the Caribbean islands, Revilla Gigedo and the Galapagos Islands, and South America down to approximately $30^{\circ}$ southern latitude. Such a wide distribution is characteristic of: two species of Lagomorpha (rabbit Oryctolagus cuniculus, cape hare Lepus capensis), three species of rodents, the roe deer, the red deer, the fallow deer, the mouflon and the wild boar.

Five of these species: the rabbit, the cape hare, the roe deer, the red deer and the fallow deer, were deliberately introduced onto the South American continent. Often these ranges are not compatible with the natural ranges of these animals, who were transported far away from their primary areas of distribution due to deliberate action by man or range expansion. Many other species were spread on other continents in the same way. The directions of their expansion and introduction are presented in Table 5. 
Tab. 5. Range changes of some species of Polish mammals; ** - successful reintroductions in recent years have increased the range of the beaver in Poland. Data source: Pucek (1984), Nowak (1971), Głowaciński (2001), Komosińska \& Podsiadło (2002).

\begin{tabular}{|c|c|c|c|c|c|c|c|c|c|}
\hline \multirow{2}{*}{ No. } & \multirow{2}{*}{ Species } & \multicolumn{2}{|r|}{ Introduction } & \multicolumn{2}{|c|}{ Invasion } & \multicolumn{2}{|c|}{ Increase in natural range } & \multicolumn{2}{|c|}{ Reduction of range } \\
\hline & & from & to & from & to & from & to & where? & how? \\
\hline 1 & 2 & 3 & 4 & 5 & 6 & 7 & 8 & 9 & 10 \\
\hline 1 & $\begin{array}{l}\text { Western } \\
\text { hedgehog }\end{array}$ & Europe & New Zealand & & & & & & \\
\hline 2 & Rabbit & & & Europe & $\begin{array}{l}\text { Australia, New } \\
\text { Zealand, North } \\
\text { America, South } \\
\text { America (Chile) }\end{array}$ & & & & \\
\hline 3 & Cape hare & Europe & $\begin{array}{l}\text { North America, South } \\
\text { America (Chile) }\end{array}$ & & & & & & \\
\hline 4 & Muskrat & $\begin{array}{l}\text { North } \\
\text { America }\end{array}$ & Europe & North America & $\begin{array}{l}\text { Europe, Asia, in all } \\
\text { directions }\end{array}$ & & & & \\
\hline 5 & Eurasian beaver & $\begin{array}{l}\text { Northern } \\
\text { Eurasia }\end{array}$ & $\begin{array}{l}\text { Poland**, Austria, } \\
\text { Germany, Switzerland, } \\
\text { France }\end{array}$ & & & & & & \\
\hline 6 & Black rat & & & South-Eeast Asia & $\begin{array}{l}\text { cosmopolitan, } \\
\text { global fauna }\end{array}$ & & & & \\
\hline 7 & Brown rat & & & North-East Asia & $\begin{array}{l}\text { cosmopolitan, } \\
\text { global fauna }\end{array}$ & & & & \\
\hline 9 & Stoat & $\begin{array}{l}\text { Palaearctic, } \\
\text { Nearctic }\end{array}$ & New Zealand & & & & & & \\
\hline 10 & Weasel & $\begin{array}{l}\text { Palaearctic, } \\
\text { Nearctic }\end{array}$ & New Zealand & & & & & & \\
\hline 11 & Western polecat & & & & & Northern Europe & $\begin{array}{l}\text { North-East } \\
\text { Europe }\end{array}$ & & \\
\hline 12 & Elk (moose) & $\begin{array}{l}\text { Eurasia, North } \\
\text { America }\end{array}$ & New Zealand & & & $\begin{array}{l}\text { Europe, Asia. } \\
\text { North America }\end{array}$ & $\begin{array}{l}\text { Europe, } \\
\text { Northern } \\
\text { Asia }\end{array}$ & & \\
\hline 13 & Red deer & Palaearctic & $\begin{array}{l}\text { North America, Argentina, } \\
\text { Australia, New Zealand }\end{array}$ & & & & & & \\
\hline 14 & Sika deer & East Asia & $\begin{array}{l}\text { Europe, Madagascar, New } \\
\text { Zealand, Australia, Japan, } \\
\text { Azerbaijan }\end{array}$ & & & & & & \\
\hline 15 & Roe deer & Eurasia & $\begin{array}{l}\text { North America, South } \\
\text { America }\end{array}$ & & & Euroasia & & & \\
\hline
\end{tabular}




\begin{tabular}{|c|c|c|c|c|c|c|c|c|c|}
\hline 1 & 2 & 3 & 4 & 5 & 6 & 7 & 8 & 9 & 10 \\
\hline 16 & Fallow deer & $\begin{array}{l}\text { Asia Minor, } \\
\text { Mesopotamia }\end{array}$ & $\begin{array}{l}\text { Europe, North America, } \\
\text { South America, Australia, } \\
\text { Oceania, New Zealand, } \\
\text { Africa, Fiji }\end{array}$ & & & & & & \\
\hline 17 & Wild boar & Europe, Asia & $\begin{array}{l}\text { North America, South } \\
\text { America, Central America, } \\
\text { New Zealand, Solomon I., } \\
\text { Tasmania, Ceylon, Sudan, } \\
\text { Australia }\end{array}$ & & & $\begin{array}{l}\text { in various } \\
\text { directions along } \\
\text { its Euroasian } \\
\text { range }\end{array}$ & & & \\
\hline 19 & Mouflon & $\begin{array}{l}\text { Corsica, } \\
\text { Sardinia }\end{array}$ & $\begin{array}{l}\text { Central Europe, Iberian } \\
\text { Pen., Crimea, NAm, SAm, } \\
\text { Hawaii, Kerguelen I. }\end{array}$ & & & & & & \\
\hline 20 & European bison & & & & & $\begin{array}{l}\text { in the course of } \\
\text { reintroduction } \\
\text { and restitution in } \\
\text { Poland and the } \\
\text { Caucasus }\end{array}$ & & & \\
\hline 22 & $\begin{array}{l}\text { European } \\
\text { souslik }\end{array}$ & & & & & & & Europe & $\begin{array}{l}\text { Reduced } \\
\text { abundance } \\
\text { throughout } \\
\text { its range in } \\
\text { Central } \\
\text { Europe }\end{array}$ \\
\hline 23 & Spotted souslik & & & & & & & Europe & \begin{tabular}{|l|} 
Disappearing \\
Polish \\
population \\
associated \\
with the \\
River Bug \\
\end{tabular} \\
\hline 24 & European mink & & & & & & & Europe & $\begin{array}{l}\text { Extinction in } \\
\text { Poland }\end{array}$ \\
\hline
\end{tabular}


There are several reasons behind these range changes. They may have been introduced into foreign habitats as game animals, one example being the wild boar (Sus scrofa), which was introduced, as described above, into North and South America and also into Australia, Tasmania and New Zealand for this purpose. This also accounts for the presence of the fallow deer, an Asian species which was introduced into Europe, and more precisely into the Mediterranean region, by the Phoenicians and later by the Romans. The Phoenicians used the fallow deer (Dama dama) as a sacrificial animal. It is currently distributed nearly all over Western and Central Europe.It was introduced into Poland as a valuable game species in the Middle Ages. Thanks to its considerable adaptability, it has also been introduced into other continents (Africa, North and South America, Australia) and some islands in Oceania (New Zealand, Fiji).

The southern chamois (Rupicapra rupicapra), a montane species, was also introduced into New Zealand. Another species associated with montane habitats, the mouflon (Ovis ammon), was also introduced as a game animal into many areas in continental Europe as well as the Americas and Hawaii.

The Polish mammalian fauna includes two completely alien species native to North America ${ }^{3}$ : the muskrat (Ondatra zibethicus) and the American mink (Mustela vison). The expansion of the muskrat across Europe and Asia over the last century is a spectacular example of a species rapidly increasing its range. A few individuals were brought to a Czech locality from Cincinnati, Ohio, in 1905. The animals escaped from captivity and expansion began in all directions at a rate of about 25 km a year. In 1924 the muskrat was seen within the Polish borders and by 1958 it had extended its range to cover the entire territory of Poland (Nowak 1971).

The other alien species, the American mink (Mustela vison), is very similar to its European cousin, the European mink (Mustela lutreola). Since 1930 it has been introduced into the British Isles, Iceland and Eastern Europe on several occasions. Some individuals also escaped from fur farms. The populations of the American mink have grown in size so much in certain areas (including Poland) that the original populations of the European mink have been pushed out of these areas (Głowaciński ed. 1992, Głowaciński ed. 2001).

The stoat (Mustela erminea), the weasel (Mustela nivalis) and the western polecat (Mustela putorius), three species of the Mustelidae family, have also increased their ranges. The stoat and the weasel have been introduced into New Zealand. Natural expansion of the western polecat (Mustela putorius) towards the north-east in Northern Europe was also observed in the previous century.

Conversely, the ranges of certain mammalian species have shrunk (Lidicker 1985). Such species include two species of souslik: the European souslik (Spermophilus citellus) and the spotted souslik (Spermophilus suslicus) (Table 5). ${ }^{4}$

There is a general decrease in the abundance of souslik populations in Eastern and Central Europe (Hoffmann et al. 2003, Afanasev 1960).

\section{Division of the total range of a species according to biogeographical units of the western and eastern Palaearctic}

The Western Palaearctic. The range of occurrence of any species is associated with certain habitat-related and biotic conditions, which vary considerably between species. In their extensive ranges, mammals occupy a variety of biotopes. Depending on habitat conditions in a given segment of the range, a taxon prefers some localities within that area. These choices

\footnotetext{
${ }^{3}$ Reports of the raccoon (Procyon lotor) penetrating into Poland are increasingly frequent now. The raccoon occurs naturally from southern Canada to Panama. It has been introduced into Europe and can be found in Germany and on the territory of the former Soviet Union.

${ }^{4}$ More examples of Polish mammals with regressive ranges are described in a later section.
} 
result in a particular distribution of the individuals, which are always found in places where the habitat conditions are within the scope of their ecological tolerance.

The 13 provinces that represent the biogeographical units of the Western Palaearctic encompass most ranges and areas of occurrence of Polish mammals (Fig. 3). Since the entire fauna of Poland belongs to the Central European Province, the ranges of the 89 species of mammals are largely confined to Central Europe. The ranges of 77 species extend further south into the Submediterranean Province, while 69 species have a part of their ranges in the Atlantic Province at the western end of the Palaearctic. The latter means that nearly $80 \%$ of the species are associated with the Atlantic shores of Western and North-Western Europe.

The ranges of 60 species are associated with the Pontic (steppe) Province of south-east Europe. This Province surrounds the Black Sea in the north and west (Fig. 3), also including the western regions of Podole, the Hungarian Lowlands, southern Moravia and the eastern part of Lower Austria.

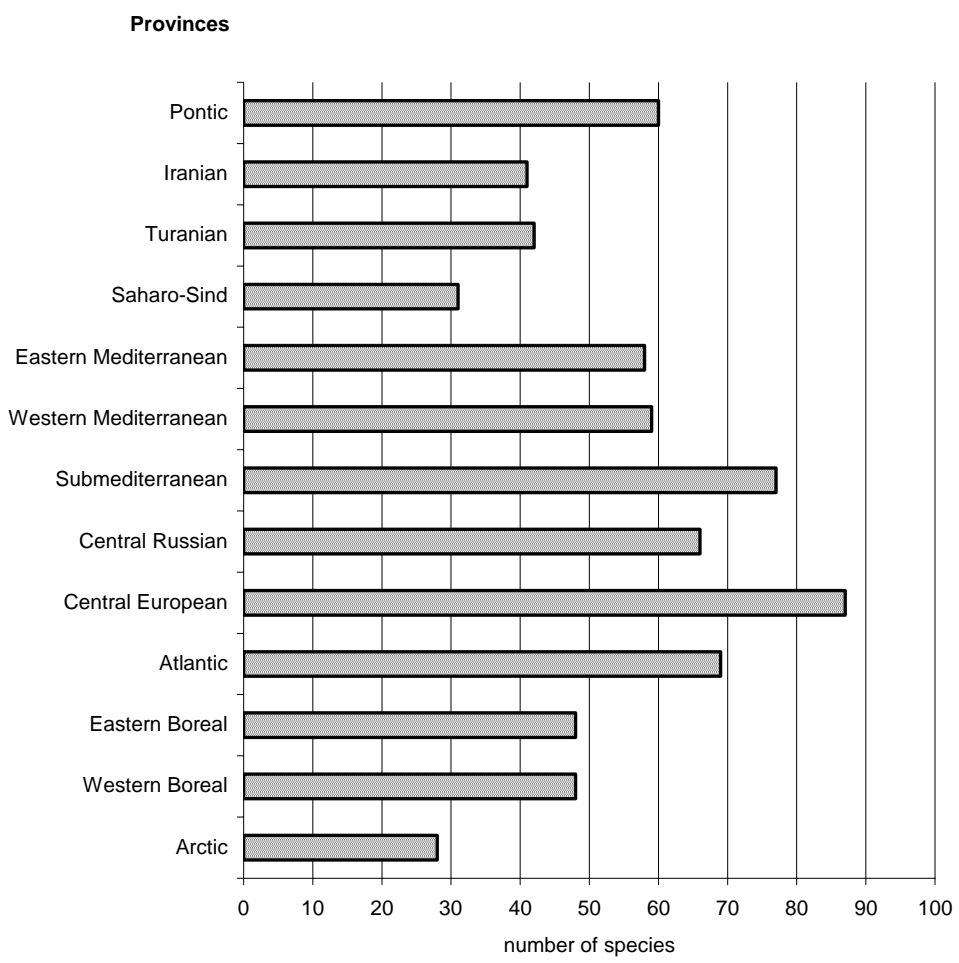

Fig. 3. Number of species associated with the provinces of the Western Palaearctic.

The ranges of 66 species extend into the Middle Russian Province, bordering Poland in the east. This means that the eastern limits of the ranges of 21 Polish mammals end in the east of Europe but do not reach into Middle Russia. This group of species comprises 3 species of shrew, 5 bat species, the rabbit (Oryctolagus cuniculus), the 2 species of souslik, the alpine marmot (Marmota marmota), the Tatra pine vole (Pitymys tatricus), the snow vole (Microtus nivalis), the pygmy field mouse (Apodemus microps), the beech marten (Martes foina), the steppe polecat (Mustela eversmanni), the fallow deer (Dama dama), the European bison (Bison bonasus), the southern chamois (Rupicapra rupicapra) and the mouflon (Ovis ammon). 
The two northern provinces of the Eurasian continent situated south of the Arctic, i.e. the Eastern and Western Boreal Provinces, support 48 species of mammals. Thus, the ranges of a half of Polish mammals extend into the northern zone of Eurasia. These two provinces are floristically much richer than the Arctic Province, with boreal coniferous forests as the prevailing type of vegetation. Ground cover is more or less developed, depending on the degree of shading of the forest floor. The proportion of dwarf shrubs, mosses and lichens increases with the severity of the climate. A large part of the zone of boreal coniferous forests is situated in the permafrost area (Mekaev 1987). The following species do not reach this far: the eastern hedgehog (Erinaceus concolor), 4 out of 8 species of shrew, 9 out of 22 species of bat, 2 species of souslik, Alpine marmot, hamster (Cricetus cricetus), Tatra pine vole, common pine vole, snow vole (Microtus nivalis), field mouse (Microtus arvalis), pygmy field mouse (Apodemus microps), garden dormouse (Eliomys quercinus), forest dormouse (Dryomys nitedula), edible dormouse (Glis glis), common dormouse (Muscardinus avellanarius), beech marten (Martes foina), steppe polecat (Mustela eversmanni), lynx (Felis lynx), wild boar, sika deer, fallow deer, European bison, southern chamois and mouflon.

Almost $70 \%$ to $90 \%$ of the species of Polish mammals can also be found in the warm and temperate climate zones of the Submediterranean, Western Mediterranean and Eastern Mediterranean provinces (Fig. 3).

Species occurring in the Irano-Turanian area account for about $50 \%$ of the Polish mammals. This area, represented in the division of the Western Palaearctic by the Iranian and Turanian Provinces, is situated in its entirety outside Europe. According to various authors, the two provinces extend from Anatolia, to the Iranian Upland, the Caspian and Turanian Lowlands, the deserts of Kara Kum and Kyzyl Kum, the Pamir, Tibet as far as the Gobi desert (Podbielkowski 1991, Formozov 1965). The Turanian and Iranian Provinces share 42 and 41 species with Poland, respectively.

The provinces sharing the least species with the area of Poland are those representing the geographical and climatic extremes, namely, the Arctic, Saharo-Sind (Saharo-Arabian) and Macaronesian provinces (Figs 3 \& 4). The Arctic province comprises Greenland, Iceland, Jan Mayen, Faroe Islands, Spitsbergen, Bear Island, Franz Josef Land, the northernmost treeless expanses of the Scandinavian Peninsula, Eastern Europe, Siberia and North America, and all islands north of these lands. The southern border of this province is the forest edge. Obviously, the extremely unfavourable climatic conditions which limit the occurrence of animals have a decisive influence on population abundance as well as species composition. The Arctic province is situated within the ranges of 28 species of mammals found in Poland.

The south-western edge of the Palaearctic is formed by the Saharo-Sind (Saharo-Arabian) and Macaronesian provinces. The former includes the Sahara, the deserts of the Arabian Peninsula, the southern edge of Belujistan and the Thar desert. The characteristics of the flora and vegetation (with deserts and semi-deserts as the prevalent plant formations) and the climatic conditions in parts of this province drastically reduce the quantitative and qualitative composition of the fauna. This province forms part of the ranges of 31 species, or $36 \%$, of the Polish mammalian fauna.

The Macaronesian Province at the south-western extreme of the Palaearctic comprises the Atlantic islands of Cape Verde, Canary Islands, Madeira and Azores. The flora of these islands is composed of a number of Mediterranean elements but there are also Boreal, Palaeotropical or even Neotropical elements. The considerable habitat diversity has resulted in the development of many different plant formations, including laurel forests, moist subtropical forests, semi-deserts and alpine plants. The mammalian fauna of this Province has 11 species in common with the mammalian inventory of Poland: the eastern hedgehog (Erinaceus concolor), 5 species of bat, the rabbit, rats, weasel and mouflon (Table 2, Fig. 4). 
The territories of three provinces: the Western Mediterranean, the Eastern Mediterranean, the Saharo-Sind, the Turanian and Iranian Province and the Macaronesian Province have been referred to as the Pramediterranean Subkingdom by some authors (Podbielkowski 1991). This subkingdom extends latitudinally from the Atlantic islands to the Mediterranean basin, North Africa and Western Asia, as far as the Gobi desert. Its flora developed on the shores of the vanishing Tetide sea and was influenced by Boreal and even East Asian floras. More tropical influences could be seen in Macaronesia and the Saharo-Sind area. There are also some associations with African and Peninsular floras that would testify to past floral exchanges between these areas. The question of faunal links between these Provinces remains open, as does the issue of present faunal exchange between these distant territories. What elements of the Polish fauna penetrate into these areas?

An analysis of faunal richness will show that provinces situated south of Poland, in southern Europe (south-western Palaearctic), differ in the number of species occurring there. There are considerable differences between, for example, the Western or Eastern Mediterranean Provinces and the Saharo-Sind or Irano-Turanian Provinces. The largest number of species that are also part of the Polish mammalian fauna can be found in two provinces of the Mediterranean basin: the Western Mediterranean and the Eastern Mediterranean Province (almost $60 \%$ of the attached list of species).

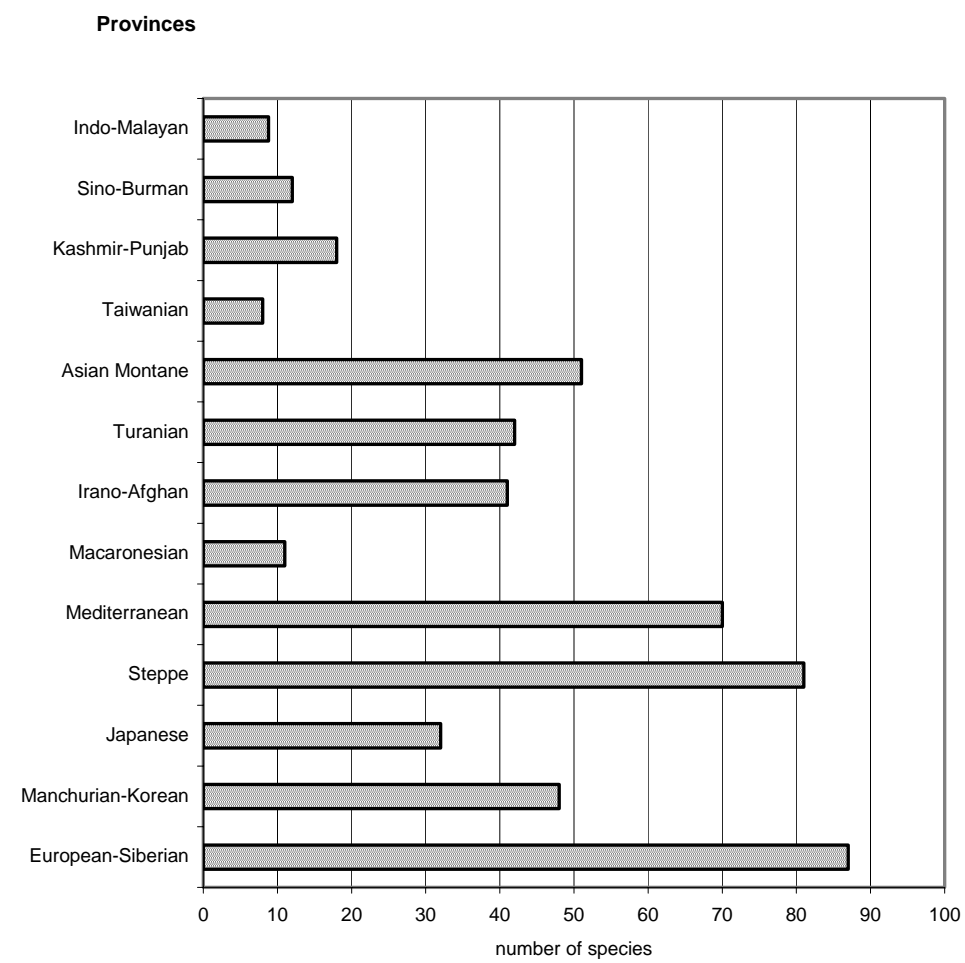

Fig. 4. Number of species associated with the provincces of the Eastern Palaearctic.

Eastern Palaearctic. This section of the paper deals with the distribution of Polish mammals in the Eastern Palaearctic Region (Fig. 2). First, the presence of species was considered with respect to the four largest units situated at the highest level of the hierarchy of 
subdivision, that is the subregions (Fig. 5). All 89 species listed as occurring in Poland can also be found in the Boreal-Eurosiberian Subregion.

The ranges of almost as many (85) species extend into the southern Mediterranean-Asian Subregion. The above findings imply that nearly all ranges $(98 \%)$ of our mammals extend (wholly or partly) to the southern limits of the Palaearctic and are associated with the Mediterranean zone and may also occupy more or less extensive areas in the Balkan Peninsula, around the Caspian Sea, in Persia, Asia Minor, Middle and Central Asia. In phytogeographical terms, these species can be regarded as a Mediterranean-Irano-Turanian "linking" element. The large number of species belonging to this element may testify to a rather close faunal relationship between these Provinces.

Finally, the Himalayan-Manchurian Subregion shares 50 mammal species with the area of Poland (Fig. 5).

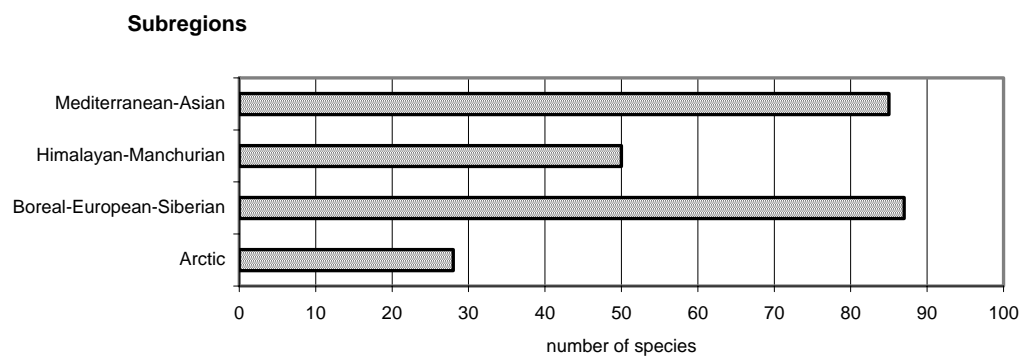

Fig 5. Number of species associated with the subregions of the Eastern Palaearctic

Figure 4 presents the numbers of mammalian species shared between the area of Poland and the Provinces of the Eastern Palaearctic, which are lower-order spatial units. A total of 13 provinces were considered. All species reach the Euro-Siberian Province.

Further east towards East Asia, Shcherbakov, who is the author of this division, distinguishes the Asian Montane province, in which he includes Gissaro-Alai, Tien-Shan and Pamir. This region shares 51 species (59\%) of mammals with the area of Poland.

The Manchurian-Korean Province in East Asia extends from the Amur in the north to the tropic of Capricorn in the south and from the upper Huang-He and the eastern slopes of the Himalayas in the west to the Korean Peninsula, Sichote-Alin, Sakhalin and the Pacific coast in the east. This province supports 48 species of Polish mammals.

The Japanese Isles are distinguished as a separate province in East Asia. This region shares 32 species with Poland: 4 species of shrew, 10 species of bat, the mountain hare (Lepus timidus), the red squirrel (Sciurus vulgaris), 5 species of rodents, the brown bear (Ursus arctos), the wolf (Canis lupus), the red fox (Vulpes vulpes), the raccoon dog (Nyctereutes procyonoides), the badger (Meles meles), the otter (Lutra lutra), the stoat (Mustela erminea), the weasel (Mustela nivalis), the American mink (Mustela vison), the wild boar (Sus scrofa) and the sika deer (Cervis nippon) (Table 1, Fig. 4).

At the lowest level of subdivision of the Eastern Palaearctic are 15 Subprovinces (Fig. 6). All species of Polish mammals occur in the Central European Subprovince and 85 can be found in Eastern Europe.

Next in the order of subprovinces ranked according to the number of species shared with Poland are the steppe subprovinces: Transcarpathian (with 70 species), Western Mediterranean, Eastern Mediterranean, Pontic and Caucasian (about 60 species). Thus, species with ranges reaching the southern part of the Palaearctic come out as most abundant (Fig. 6). 
A similar number of species is shared between the Danubian Subprovince and the WesternSiberian Subprovince (Fig. 6).

\section{Subprovinces}

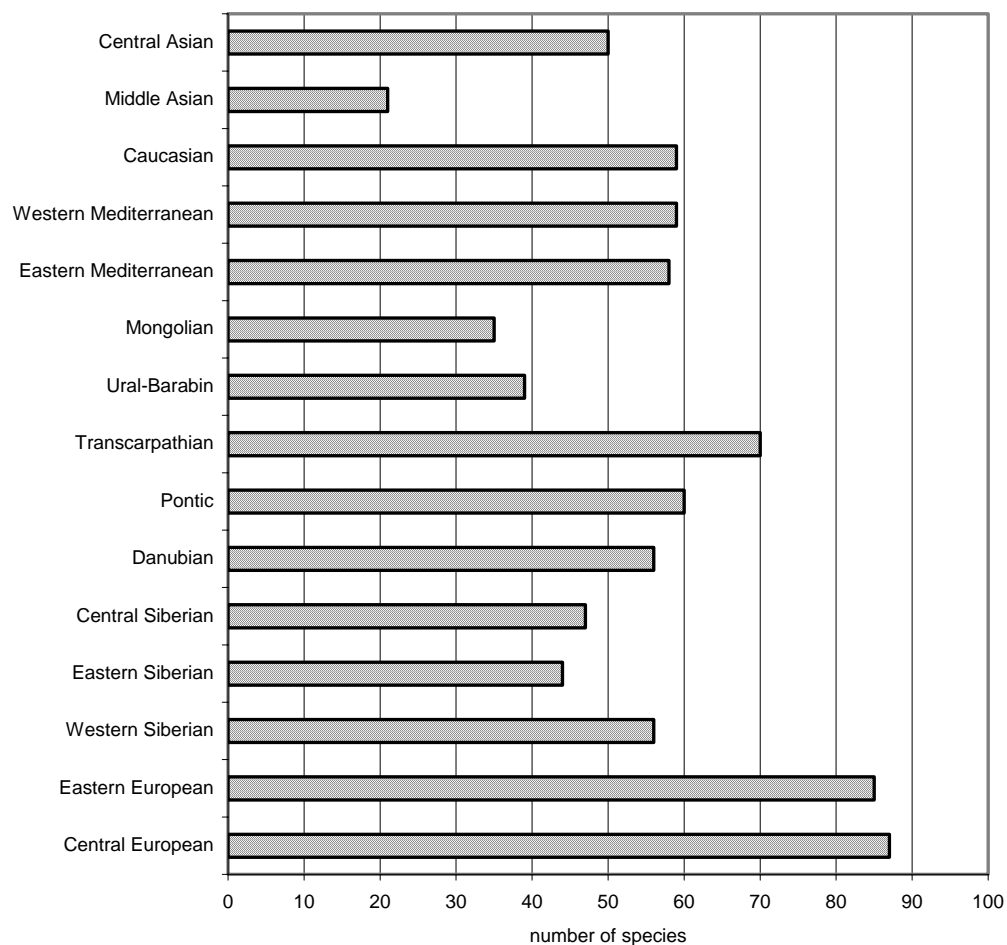

Fig. 6. Number of species associated with the subprovinces of the Eastern Palaearctic.

The Western Siberian, Central Siberian and Central Asian Subprovinces support a slightly smaller number of Polish species (44-50) with 21 species reaching the Central Asian Montane Subprovince.

\section{Changes in natural range boundaries due to anthropogenic impacts}

When discussing the natural boundaries of Polish mammals' ranges, it has to be emphasised that some of them have been modified, especially in the $20^{\text {th }}$ century. These changes are the result, on the one hand, of the retreat of species from some areas and a decline of their populations and, on the other, of the appearance of new alien species that are expansive and thus represent a threat to the indigenous taxa. These two processes are reflected in the dynamics of range development. Some changes are anthropogenic, due to the introduction of alien species into previously unoccupied areas. One can say that the initial ranges of some species have changed owing to the penetration of the species into new territories. Accordingly, secondary ranges need to be listed for many species which are the result of the species spreading over increasingly larger areas. The descriptions of species ranges presented in this paper together with their divisions according to the accepted system of zoogeographical units determine their extent in the Palaearctic. The ranges of most Polish mammals are confined to Europe and Asia. Many have changed due to anthropogenic impacts (Table 5). 
Changes in species ranges are also the result of the introduction or reintroduction of species into new areas. Introduced species can be divided into two groups. One includes those species which were introduced into nature in other countries and have spread into Poland in the course of their subsequent expansion on the European continent. The other group consists of species introduced directly into Poland (Andrzejewski \& Pielowski 2003). There are several examples of successful introductions or reintroductions of mammals in Poland (Table 5):

1. The fallow deer (Dama dama) whose original range extended over the Mediterranean regions of Europe, Africa and the Middle East as far as Iran, has been acclimatised in various parts of our continent, which has resulted in a secondary expansion of its range. The fallow deer was introduced into Poland as early as $17^{\text {th }}$ century and now occurs in multiple small-sized populations. It has also been introduced onto other continents: North and South America, Australia, Oceania, New Zealand, Africa and the island of Fiji.

2. The sika deer (Cervus nippon) originally inhabited East Asia. It has been acclimatised in many European countries, including Poland (twice in the 19th and 20th centuries). At present there are two local micropopulations in Poland showing no tendency to migrate. In its secondary range, the sika deer is also beginning to spread to other continents and areas, such as Madagascar, New Zealand, Australia, Azerbaijan and Japan.

3. The mouflon (Ovis ammon) could originally be found only on the islands of Corsica and Sardinia. In the $19^{\text {th }}$ and early $20^{\text {th }}$ centuries, mouflons were introduced into numerous faunal refuges in Central Europe, the Iberian Peninsula and the Crimea as well as in the U.S.A. (California, Texas), South America, Hawaii and the Kerguelen Islands. Mouflons were first introduced into Poland in 1901. At present they occur in the Sudetes region.

4. The muskrat (Ondatra zibethicus) spread to Poland in the course of its invasion. It had been brought into Czech territory from North America for breeding on fur farms. Having escaped from captivity, the muskrat was first seen on Polish territory as early as 1924 and subsequently reached high densities characteristic of an invading species in the 1930's and 1940's. The muskrat has thus become a component of the European and Polish fauna, extending its natural North American range to other continents (Europe and Asia).

5. The eurasian beaver (Castor fiber) was an endangered species in Poland until quite recently, surviving only in residual populations in the north-east of the country. At present the populations have been restored and transferred to various sites across the country and the species is no longer under direct threat of extinction. Its range in Europe has been artificially extended in Germany, Switzerland and France and the beaver is now expanding its Eurasian distribution.

6. The raccoon dog (Nyctereutes procyonoides) - the natural range of this canine predator is in east Asia, from Manchuria and Ussuri to China and Japan, to Indochina. It spread to Poland from ex-Soviet republics, where it had been acclimatised since the 1930's. The raccoon dog was first encountered in Poland in 1955 and has since then become abundant in forests, especially along rivers and lakes. At present it occurs in the whole of Europe and Central Asia. A new, large secondary range of this species was later formed in Europe through planned introduction over a period of 30 to 40 years and a couple of new sites were established in Central Asia in the same way. At the same time, natural spread of the species occurred and both these processes led to the development of a new continuous range in the western part of the former Soviet Union in the early 1960's. Raccoon dogs were first seen in eastern Poland at two locations in 1955. In 1962 and 1963 raccoon dogs were traced and shot in the west and south of Poland. The Atlas of Distribution of Mammals in Poland (Pucek \& Raczyński, 1983) shows sites where the raccoon dog has been found all over Poland.

7. The American (Mustela vison) mink was introduced into Europe in the first half of the $20^{\text {th }}$ century for breeding in fur farms. Its spread into the wild followed its escape from captivity. 
The species appeared in Poland in the 1960's. The American mink is considered to have ousted the European mink from Poland to complete extinction, through rapid invasion and spread into aquatic habitats. The American mink has expanded its original range from a North American one to a Eurasian one, with the formation of a secondary range of the in the wild in Europe and Asia. At the same time the range of the European mink in Europe and Asia has shrunk. The European mink disappeared from the area of Poland in the $20^{\text {th }}$ century. According to research data from specialists in this species, it was last seen in the 1930's.

Range reduction may also be seen over time, apart from range expansion. Three species whose ranges have decreased in Europe or which have completely disappeared from Poland have already been mentioned. These are: the European mink, the European souslik and the spotted souslik. More examples of population decrease and reduction in the area of range among Polish mammals can be provided. While data regarding range dynamics in the world are usually lacking, there is evidence pointing to the threat of extinction of those species and the need to protect them.

1. The garden dormouse (Eliomys quercinus) is the most critically endangered rodent species in Poland. It occurs in small isolated populations. There are data suggesting decreasing abundance of the garden dormouse both in Poland and in the neighbouring countries. As the current distribution of this species in Poland is poorly known, it is difficult to determine whether the range is generally regressive.

2. The forest dormouse (Dryomys nitedula) is a Eurasian species with a rather large insular distribution that reaches its north-western limit in Poland. While no definitive statements can be made, it can be supposed that the partitioning of the area occupied by this species along the north-western limit of its range on Polish territory is associated with the possibility of extinction of the resulting minute isolated populations.

3. The spotted souslik (Spermophilus suslicus) is a relic species of Pontic origin whose range covers Central and Eastern Europe. It has been present in Poland for a long time, occupying a stable territory between the Rivers Wieprz and Bug. Its abundance has been decreasing and there is a dwindling enclave relic population in the Zamość region (south-east Poland). This species inhabits steppe areas. In 1953-1954 it was registered at 143 sites with a total population of 70,000. Since then the population size has been constantly decreasing and the area occupied by the species has been reduced by more than a half of its original size (Głowaciński ed., 2001, Pucek 1989). The westernmost limit of the range is east Poland and the border between Russia and north-east Rumania.

4. The European souslik (Spermophilus citellus) is a Eurasian species that reaches Manchuria and Korea at the south-eastern tip of its range. This species has died out in its only relic refuge in Poland, in the Opole region [in the south west], which marked the northern limit of its range. The abundance of this species is rapidly falling throughout its range, which is mostly due to habitat change. Regrettably, the last record of the European souslik in Poland dates back to 1973, with later data not containing any mention of finding a European souslik individual or traces of its activity in Poland. It is therefore considered extinct. Some individuals are known to have survived in Saxony until the 1980's. Apart from central and eastern Europe, the European souslik is distributed throughout East Asia as far as China.

5. The alpine marmot (Marmota marmota) is an alpine species with a range confined to two populations in the Alps and Tatra Mountains. The Tatra population numbers 150-200 individuals. A slight increase in population size has been seen over the last half of the century, but high annual variations and the stopping of expansion of the territory occupied by this species still place it among species at high risk of extinction.

6. The southern birch mouse (Sicista subtilis) is a Eurasian species which was first encountered in Poland in 1994 at one site in the south-east. The range changes and 
development dynamics of this rather rare species are not clear. It has been listed as an extinct species in Austria.

7. The edible dormouse (Glis glis) is another Eurasian species. studies in north-western Poland (Pomerania, Great Poland [Wielkopolska], Ziemia Lubuska [Lubus Land]) in the 1990's revealed that the edible dormouse was extinct at most sites there. There is a lack of detailed data on the development of populations in the world. It has been declared a protected species, close to extinction and is protected under the Berne Convention. It is also found on the Red List of IUCN.

8. The southern chamois (Rupicapra rupicapra) is a montane European and West Asian species occupying several disjunct areas in the European mountains (Cantabrian Mts., Pyrenees, Alps, Apennines, Balkan ranges, Carpathians) and the Caucasus and mountain ranges in Asia Minor. The Tatra chamois, which reaches the northern limit of its range in Poland, is a critically threatened species, found on the IUCN Red List. It is legally protected pursuant to the Berne Convention.

Species formerly regarded as common (cape hare Lepus europaeus and hamster Cricetus cricetus) are now listed among threatened species.

\section{CONCLUSIONS}

1. Taxonomically, the Polish mammalian fauna includes species belonging to 20 families, whose ranges extend well beyond the European borders.

2. The ranges of all species of Polish mammals are located in the Palaearctic, with some extending into other biogeographical regions.

3. Some Polish mammals are distributed on almost all continent. The rabbit (Oryctolagus cuniculus), cape hare (Lepus capensis), brown rat (Rattus norvegicus), black rat (Rattus rattus), red deer (Cervus elaphus), elk (Alces alces), wild boar (Sus scrofa) or the mouflon (Ovis ammon) are species with wide ranges and can consequently be regarded as elements of the 'fauna of the global continent'.

4. Many Polish mammal species have changed the size of their ranges through introduction, expansion, invasion or due to the effect of natural factors rather than man's purposeful activity. Some of the species have developed secondary (artificial, anthropogenic) ranges which are sometimes situated at a considerable distance from their natural ranges. Such range changes due to natural factors (active) or mediated by man have been demonstrated for the rabbit (Oryctolagus cuniculus), muskrat (Ondatra zibethicus), wild boar (Sus scrofa), raccoon dog (Nyctereutes procyonoides), stoat (Mustela erminea), western polecat (Mustela putorius), weasel (Mustela nivalis), American mink (Mustela vison), elk (Alces alces), roe deer (Capreolus capreolus), red deer (Cervus elaphus) and sika deer (Cervus nippon).

5. The ranges of about $60-80(67 \%-90 \%)$ Polish mammals extend into Palaearctic provinces in the warm climate zone: Submediterranean, Eastern Mediterranean, Western Mediterranean, Atlantic, Pontic.

6. There are mammalian species whose ranges have decreased to the point of complete extinction (European mink Mustela lutreola, European souslik Spermophilus citellus).

7. The range of 28 (approx. 32\%) Polish mammals extend into the coldest areas within the Palaearctic (Arctic Subregion).

8. The Provinces constituting the south-western limit of the Palaearctic (Macaronesia, Saharo-Sind Province) are penetrated by $11(12 \%)$ and 31 (35\%) Polish mammal species, respectively.

9. The steppe Provinces of the Palaearctic (Danubian, Pontic, Transcarpathian, UraloBarabin, Mongolian) share 81 (91\%) of mammals with Poland. 
10. The Asian provinces (Turanian, Irano-Afghan and Asian Montane) are penetrated by about $40-50(45 \%-56 \%)$ of the species, with the greatest number found in the Asian Montane Province).

\section{REFERENCES}

AFANASEV A. V. 1960. Zoogeografiya Kazakhstana. Na osnove razprostranienia mlekopitayushchikh. Izdatel'stvo Akademii Nauk Kazakhskoï U.S.S.R. 246 pp. [In Russian]

ANDRZEJEWSKI R. \& PIELOWSKI Z. 2003. Gospodarka łowiecka a różnorodność biologiczna. In: ANDRZEJEWSKI R. \& WeIGle A. (eds), Różnorodność biologiczna Polski, pp. 217-223. Narodowa Fundacja Ochrony Środowiska, Warszawa, $284 \mathrm{pp}$.

Corbet G. B. 1978. The Mammals of the Palaearctic Region: a taxonomic review. British Museum (Natural History). Cornell University Press, London, 298 pp.

Corbet G. B. \& Hill J. E. 1991. A world List of Mammalian Species. 3rd Edition. Natural History Museum Publications. Oxford University Press, Oxford, $232 \mathrm{pp}$.

Formozov A.N. (ed.) 1965. Mlekopitayushchie U.S.S.R. Izdatel'stvo 'Mysl', Moscow, 438 pp. [In Russian]

GentNer V. G., NASIMOVICH A. A. \& BANNIKOV A. G. 1961. Mlekopitayushche U.S.S.R.. Otryad parnokopytnykh i neparnokopytnykh. Ordo Artiodactyla (Owen, 1848). Vol. I -III. Gosudarstvennoe izdatel'stvo 'Vyzhshaya shkola', Moscow, 776 pp. [in Russian]

GŁowAciński Z. (ed.) 1992. Polish Red Data Book of Animals. Państwowe Wydawnictwo Rolnicze i Leśne, Warszawa, $352 \mathrm{pp}$.

GŁowACIŃSKI Z. (ed.) 2001. Polish Red Data Book of Animals. Vertebrates. Państwowe Wydawnictwo Rolnicze i Leśne, Warszawa, $452 \mathrm{pp}$.

GÖRneR M. \& HACKethal H. 1987. Säugetiere Europas. Beobachten und bestimmen. Neumann Verlag Leipzig, Radebeul, $364 \mathrm{pp}$.

GOSZCZYŃSKI J. 1995. Lis. Monografia przyrodniczo-łowiecka. Oikos, Warszawa, 137 pp.

GoszcZyŃSKi J., RomAnOwSKI J. \& Zalewski A. 1994. Kuny. Oficyna Edytorska „Wydawnictwo Świat”, Warszawa, $62 \mathrm{pp}$.

Gromova N. M. \& BARANOvoi G. I. (eds). 1981. Katalog mlekopitayushshchikh U.S.S.R. 'Nauka' .Acad.. Sci. U.S.S.R.. Zool. Inst., St. Petersburg, 455 pp. [in Russian]

Hoffmann I. E., Millesi E., Pieta K. \& DitTAmi J. P. 2003. Anthropogenic effects on the population ecology of European ground squirrels (Spermophilus citellus) at the periphery of their geographic range. Mammalian Biology 68: 205-213.

KomosińSKA H. \& PODSIAdŁo E. 2002. Ssaki kopytne. Przewodnik. Wydawnictwo Naukowe PWN, Warszawa, 304 pp.

LIDICKER W. Z. (ed.) 1985. Rodents. A World of Species of Conservation Concern. Occasional Papers of the IUCN Species Survival Commission (SSC). No. 4 . IUCN- pp. 26-32. The World Conservation Union, Edmonton, Alberta , Canada.

KoRNAŚ J. \& MEDWECKA-KoRNAŚ A. 2002. Geografia roślin. Wydawnictwo Naukowe PWN, Warszawa, 614 pp.

MEKAEV J. A. 1987. Zoogeograficheskie kompleksy Evrazii . Izdatel'stvo 'Nauka', St. Petersburg, 125 pp. (in Russian)

Mitchell-Jones A. J., Amori G, Bogdanowicz W., KryštufeK B., Reijnders P. J. H., Spitzenberger E., Stubbe M., Thissen J. B. M., Vohralik V. \& Zima J, 1999. The atlas of European mammals. Acadaemic Press, London, $482 \mathrm{pp}$.

NiETHAMMER J. \& KRAPP F. 1986. Handbuch der Säugetier Europas. Aula-Verlag, Wiesbaden, 384 pp.

NowAK E. 1971. The Range Expansion of Animals and its Causes. Polska Akademia Nauk . Instytut Ekologii. Zeszyty Naukowe, Warszawa, 3: 252 pp. [In Polish]

PoDBIELKOWSKi Z.1991. Geografia roślin. Wydawnictwa Szkolne i Pedagogiczne, Warszawa, 519 pp.

PuCEK Z. (ed. ). 1984. Klucz do oznaczania ssaków Polski. PWN, Warszawa, 386 pp.

PuCEK Z. 1989. A Preliminary Report on Threatened Rodents in Europe. Rodents. A World Survey of Species of Conservation Concern. In: LIDICKER W Z. Jr. (ed.), Occasional Papers of the IUCN Species Survival Commission (SSC) 4: 26-32.

PuCEK Z. \& RACZYŃSKi J. 1983. Atlas rozmieszczenia ssaków w Polsce. PWN, Warszawa,183 pp.

SyroechKovskil̈ E. E. \& Rogacheva Ė. V. 1975. Zhivotnyï Mir U.S.S.R.. Izdatiel'stvo 'Mysl', Moscow, 440 pp. [In Russian]

SZAFER W. \& ZARZYCKI K. (eds). 1972. Szata roślinna Polski.. 2nd Edition., Vol. I. \& II. PWN, Warszawa, $615+$ $342 \mathrm{pp}$.

SzCZERBAK N. N. 2003. Guide to the Reptiles of the Eastern Palaearctic. Krieger Publishing Company. Malabar, Florida, 252 pp.TUPIKOVA N. V. 1969. Zoologicheskoe kartografirovanie. Izdatel'stvo Moskovskovo Universiteta, Moscow, 250 pp. [in Russian]

UdVARDY M. D. 1969. Dynamic zoogography. Van Nostrand Reinhold Company, New York, 446 pp. 
Wilson E. D. \& ReEder D. A. 1993. Mammals species of the World. A taxonomic and Geographic Reference. 2nd Edition. Smithsonian Institution Press. Washington and London in association with the American Society of Mammologists. (SSC). No. 4 . IUCN- The World Conservation Union. Edmonton, Alberta , Canada, 1002 pp.

WolosZYN B. W. 2001. Bats of Poland. Distribution, habitat and conservation status. Publication of the Chiropterological Information Center. Institute of Animal Systematics and Evolution. Polish Academy of Sciences, Kraków, 86 pp.

\section{STRESZCZENIE}

\section{[Zasięgi ssaków Polski na tle jednostek zoogeograficznych Palearktyki]}

Kształt i wielkość areałów poszczególnych gatunków oraz ich zmiany są podstawą wszelkich wnioskowań natury biogeograficznej. Etapem poprzedzającym to wnioskowanie powinien być opis pełnego zasięgu umiejscawiający go w przyjętym podziale zoogeograficznym. Szczególnie interesujące wnioski można uzyskać, analizując powiązania zasięgów z konkretnymi terytoriami na kuli ziemskiej. Dotychczas nie opracowano całościowych map zasięgów dla wszystkich gatunków kręgowców, oddających zarówno strukturę wewnętrzną zasięgu jak i jego zarys ogólny. W niniejszej publikacji opisano pełne zasięgi każdego z 89 gatunków ssaków lądowych występujących w Polsce, co daje podstawę do dalszych analiz zoogeograficznych. Przedstawiona klasyfikacja zoogeograficzna posłużyć może również do ustalania korelacji między zasięgami roślin i zwierząt a przestrzennym zróżnicowaniem czynników środowiskowych, w efekcie do konstruowania podziałów regionalnych opartych na kryteriach florystycznych, faunistycznych i zróżnicowaniu geograficznym zasięgów. 